\title{
Polystyrene Backbone Polymers Consisting of Alkyl-Substituted Triazine Side Groups for Phosphorescent OLEDs
}

\author{
Beatrice Ch. D. Salert, ${ }^{1}$ Armin Wedel, ${ }^{1}$ Lutz Grubert, ${ }^{2}$ Thomas Eberle, ${ }^{3}$ \\ Rémi Anémian, ${ }^{3}$ and Hartmut Krueger ${ }^{1}$ \\ ${ }^{1}$ Department of Polymers and Electronics, Fraunhofer Institute for Applied Polymer Research, \\ Geiselbergstraße 69, 14476 Potsdam, Germany \\ ${ }^{2}$ Department of Chemistry, Humboldt University, Brook-Taylor-Straße 2, 12489 Berlin, Germany \\ ${ }^{3}$ Merck KGaA, Frankfurter Straße 250, 64293 Darmstadt, Germany \\ Correspondence should be addressed to Hartmut Krueger, krueger@iap.fhg.de \\ Received 9 March 2012; Accepted 16 April 2012 \\ Academic Editor: Etienne Baranoff
}

Copyright ( $) 2012$ Beatrice Ch. D. Salert et al. This is an open access article distributed under the Creative Commons Attribution License, which permits unrestricted use, distribution, and reproduction in any medium, provided the original work is properly cited.

This paper describes the synthesis of new electron-transporting styrene monomers and their corresponding polystyrenes all with a 2,4,6-triphenyl-1,3,5-triazine basic structure in the side group. The monomers differ in the alkyl substitution and in the meta-/ paralinkage of the triazine to the polymer backbone. The thermal and spectroscopic properties of the new electron-transporting polymers are discussed in regard to their chemical structures. Phosphorescent OLEDs were prepared using the obtained electrontransporting polymers as the emissive layer material in blend systems together with a green iridium-based emitter $\mathbf{1 3}$ and a small molecule as an additional cohost with wideband gap characteristics $(\mathrm{CoH}-001)$. The performance of the OLEDs was characterized and discussed in regard to the chemical structure of the new electron-transporting polymers.

\section{Introduction}

In 1990, when Burroughes et al. published first results on light-emitting diodes based on fluorescent conjugated polymers, these materials achieved more and more interest not only in science but also in technical application in displays and lighting [1]. From this time onwards, the development of full color flat panel displays was a main focus in research and development. Those displays would gain many advantages such as a wide viewing angle, high brightness and processing on flexible substrates. This is due to the ability of easy solution processing for large areas via different printing techniques like ink-jet printing [2] or roll-to-roll [3] procedures. During the past ten years, research activities have been more and more focused on phosphorescent systems. One reason for this is their lower power consumption [4]. Higher quantum efficiencies can be obtained in a light-emitting diode using phosphorescent transition metal complexes containing organic ligands instead of singlet-emitting materials [5]. Some transition metal complexes, particularly iridium-(III) complexes, show very short triplet excited state lifetimes due to high radiative decay rates. Up to $100 \%$ internal quantum efficiencies of these phosphorescent emitters are possible in adapted host systems [6].

Solution-processable phosphorescent materials can be broken down into two categories-solution based small molecules and polymers. Both material approaches consist of a transition metal complex acting as an efficient emitter and of a charge transport host responsible for an efficient hole and electron transport to the emitter. Suzuki et al. [7] introduced polystyrene as nonconjugated polymer backbone carrying the emitter and the host units as covalent attached side groups. As it is known polystyrene is an optically and electrically stable backbone. Due to this fact, polystyrene can be used as the polymer main chain while substitutions of several active side groups can be investigated.

Following this idea, we recently introduced some novel hole- and electron-transporting units as side groups of a polystyrene backbone and tested them in phosphorescent 
devices [8-10]. To improve and to adapt the electron transport in phosphorescent systems for small-moleculebased OLEDs tris-(8-hydroxyquinoline) aluminum $\left(\mathrm{AlQ}_{3}\right)$ is a very commonly used electron transporting material. First reports using this material were published about 25 years ago [11]. During the past five years, research is looking for alternative electron-transporting materials. Chen et al. and Inomata et al. studied 1,3,5-triazine-core structures and their behavior in OLEDs. They used different substituted 1,3,5triazine core structures as small molecules in OLEDs $[12,13]$. Klenkler et al. recently published a triazine-based structure which shows high electron mobility resulting in OLEDs with a lower driving voltage and higher efficiency [14]. Behl and Zentel [15] studied first p-vinyl-substituted 2,4,6-tripheny1,3,5-triazine monomers, their polymerization, and the OLED performance using the obtained polymers as electrontransporting matrices.

In this paper, we now introduce several new electrontransporting units all based on a central 2,4,6-triphenyl1,3,5-triazine unit. According to Chen et al. and Inomata et al. [12,13], who studied 1,3,5-triazine-core structures as small molecules, we decided to combine the idea of those groups to the studies done by Suzuki et al. [7] using polystyrene as a nonconjugated polymer backbone. Besides analyzing a simple nonsubstituted 1,3,5-triazine core polymer, we wanted to get more information about OLED performance for substituted polymers. As substitution pattern we chose several small alkyl substituents and compared their properties with their nonsubstituted analogues in OLEDs. Therefore, several new styryl derivatives with different dialkyl substituents to the triazine core were synthesized and polymerized. The polymers were characterized with UV-Vis- and fluorescence spectroscopy and with DSC. Cyclovoltammograms of their monomers were discussed to describe the energy levels of their corresponding polymers. The influence of different 2,4,6-substitutions of the triazine core structures for the OLED performance was studied by using them as electron-transporting components. Therefore, blends of the new triazine containing polystyrenes with an iridium-tris-2-phenylpyridine derivative $\mathbf{1 3}$ as phosphorescent green emitter and a small molecule $\mathrm{CoH}-001$ (from Merck) as an additional cohost with wideband gap characteristics were prepared. From the blends, OLEDs were built up, and the results are compared in regard to brightness, electroluminescence, and efficiency.

\section{Experimental}

2.1. Synthesis and Methods. All chemicals were obtained from Merck KGaA, Fluka or Sigma Aldrich and were used without further purification. All synthesis steps were carried out under argon atmosphere, and dry solvents were used which were stored over a molecular sieve. Silica gel (60 Merck) was used for column chromatography. The polymerizations were carried out in a glove box under argon atmosphere, and tetrahydrofuran (freshly distilled) was used. OLED device preparation was carried out in a glove box.
2.1.1. High Resolution with TMS as Standard NMR Spectra. ${ }^{1} \mathrm{H}-\mathrm{NMR}$ and ${ }^{13} \mathrm{C}-\mathrm{NMR}$ spectra of the synthesized intermediates, monomers, and polymers were measured with a UNITY INOVA 500 Spectrometer from Varian. As solvents, deuterated chloroform or tetrahydrofuran was used.

Elemental analyses were obtained by using a Thermo Scientific FlashEA 1112 CHNS/O Automatic Elemental Analyser.

2.1.2. Gel Permeation Chromatography. To determine the molecular weights and their distribution of the synthesized polymers, a combined system consisting of a water HPLC pump 515, autosampler 717 plus, dual $\lambda$ absorption detector 2487, and a refraction index detector 2414 was used. The polymers were measured in tetrahydrofuran at room temperature. A precolumn and three columns from Waters $(7,8 \mathrm{~mm} \times 300 \mathrm{~mm}$; Styragel HR3, HR4, HR5) filled with a copolymer of styrene and divinylbenzene $5 \mu \mathrm{m}$ and PS standards from Polymer Laboratories were used. Polymer solutions of $2 \mathrm{mg} / \mathrm{L}$ in tetrahydrofuran were used which were stirred at room temperature for 24 hours before they were filtered through a syringe filter $(1 \mu \mathrm{m})$ and measured.

Thermal analysis was obtained by using a Netzsch DSC 204 system with a scan rate of $10 \mathrm{~K} / \mathrm{min}$. About $5 \mathrm{mg}$ of the polymer was weighed in a $40 \mu \mathrm{L}$ aluminum pan.

2.1.3. UV/VIS Spectroscopy. The synthesized polymers were measured as a film on silica glass. The films were prepared from chloroform solution $(7,5 \mathrm{mg} / \mathrm{mL})$ by spin coating. For the measurement, a Carry 5000 UV/VIS Spectrometer of Perkin-Elmer was used.

2.1.4. Photoluminescence Spectroscopy. The same films that were used for UV-Vis measurements were measured at a Perkin-Elmer LS50B to determine photoluminescence. The excitation wavelength was $300 \mathrm{~nm}$.

2.1.5. Cyclovoltammetry. Cyclic voltammetry was performed using a PG310 USB (HEKA Elektronik) potentiostat interfaced to a PC with PotMaster v2x43 (HEKA Elektronik) software for data evaluation. A three-electrode configuration contained in a nondivided cell consisting of a platinum disc $(d=1 \mathrm{~mm})$ as working electrode, a platinum plate as counter-electrode, and a saturated calomel electrode (SCE) with an agar-agar-plug in a Luggin capillary with a diaphragm as reference electrode was used. Measurements were carried out in $\mathrm{N}, \mathrm{N}$-dimethylformamide (anhydrous, SIGMA-ALDRICH) containing $0.1 \mathrm{M} \mathrm{Bu}_{4} \mathrm{NPF}_{6}$ (FLUKA) using a scan rate of $\mathrm{d} E / \mathrm{d} t=1 \mathrm{~V} \mathrm{~s}^{-1}$. The data is given in reference to the ferrocene redox couple $\left(\mathrm{Fc} / \mathrm{Fc}^{+}\right)$which was used as external standard.

2.1.6. OLED Preparation. For the OLED preparation, ITOcoated glass substrates were used which were obtained from Optrex Europe $\mathrm{GmbH}$ (sheet resistance $20 \mathrm{Ohm} / \square$; luminance area $=4 \mathrm{~mm}^{2}$ ). Before preparation, the ITOglass substrates were cleaned via ultrasonic bath. The first hole injection layer was applied by spin-coating 
a water-based dispersion of polyethylenedioxythiophene/ polystyrene sulfonic acid (Clevios CH8000; Heraeus Holding $\mathrm{GmbH})$. The layer was dried at $130^{\circ} \mathrm{C}$ for five minutes. As a hole-transporting layer, Interlayer 1 (Merck KGaA) was coated on the substrate. This is followed by a spin-coating process of the emitting polymer blend which was dissolved in toluene for 24 hours and filtered through a syringe filter with pore size of $0,2 \mu \mathrm{m}$ before preparation. The blend consists of emitter 13, the wideband gap small molecule $\mathrm{CoH}$ 001 and the electron-transporting polymer $\mathbf{9 a} / \mathbf{b}-\mathbf{1 2} \mathbf{a} / \mathbf{b}$. The wideband gap small molecule and the electron-transporting polymer $\mathbf{9 a} / \mathbf{b}-\mathbf{1 2} \mathbf{a} / \mathbf{b}$ were added with $41,7 \mathrm{wt} \%$ while the emitter 13 was added with $16,7 \mathrm{wt} \%$.

After the spin-coating process, another drying process was done at $110^{\circ} \mathrm{C}$ for 10 minutes to completely remove the solvent. The cathode $(5 \mathrm{~nm} \mathrm{Ba}$ and $150 \mathrm{~nm} \mathrm{Al})$ was deposited by a thermal evaporation process under high vacuum. The preparation was done in a glove box under nitrogen. For OLED characterization, luminance-voltage plots, efficiency-voltage plots, and electroluminescence spectra were measured with a Konica Minolta Camera CS2000. The electroluminescence was measured with a Diode-Array Spectrometer EPP 2000 from Stella Net. Inc.

\subsubsection{Monomer Synthesis}

2-(3-Bromo-Phenyl)-4,6-Diphenyl-[1,3,5]-Triazine $\left(\mathbf{3} \mathbf{a}, \mathbf{R}_{\mathbf{1}}=\right.$ H). $60,59 \mathrm{~g}(0,454 \mathrm{~mol})$ aluminium trichloride was exhibited. After the addition of $9,9 \mathrm{~mL}(0,136 \mathrm{~mol})$ thionylchloride, $60 \mathrm{~mL} \quad(0,454 \mathrm{~mol})$ 3-bromobenzoylchloride, and $98,4 \mathrm{~mL}$ benzonitrile, the chemicals were dissolved in $800 \mathrm{~mL}$ 1,2-dichlorobenzene. The mixture was stirred for 20 hours at $100^{\circ} \mathrm{C}$. After 1 hour, 48,61 g $(0,909 \mathrm{~mol})$ ammonium chloride was added to the reaction. After cooling to room temperature, the reaction mixture was poured into $3 \mathrm{~L}$ methanol and stirred for 45 minutes. The solid product was filtered and washed with hot ethanol. After drying under reduced pressure at $60^{\circ} \mathrm{C} 36,69 \mathrm{~g}$ (20\% yield), a solid white product was obtained.

${ }^{1} \mathrm{H}-\mathrm{NMR}\left(500 \mathrm{MHz}, \mathrm{THF}-\mathrm{d}_{8}, \delta\right): 8,93$ (s, $1 \mathrm{H}$, aromatic); $8,80(\mathrm{~d}, 5 \mathrm{H}$, aromatic, $J=6,83) ; 7,80(\mathrm{~d}, 1 \mathrm{H}$, aromatic, $J$ $=7,08) ; 7,59(\mathrm{~m}, 6 \mathrm{H}$, aromatic $) ; 7,53(\mathrm{t}, 1 \mathrm{H}$, aromatic, $J=$ $8,06)$.

2,4-Diphenyl-6-(4'-Vinyl-Biphenyl-3-yl)-[1,3,5]-Triazine (5a). $1,16 \mathrm{~g}(0,003 \mathrm{~mol})(3 \mathbf{a}), 0,74 \mathrm{~g}(0,005 \mathrm{~mol}) 4$-vinylphenylboronic acid (4) and 0,02 $\mathrm{g}(0,02 \mathrm{mmol})$ tetrakis(triphenylphosphine)palladium(0) were dissolved in $45 \mathrm{~mL}$ THF, and after the addition of $30 \mathrm{~mL} 1 \mathrm{M}$ sodium carbonate solution, the mixture was stirred for 24 hours at reflux. After cooling to room temperature, the mixture was filtered over celite, and diluted with ethyl acetate. The mixture was washed with water three times, and the solvent of the organic layers was removed under reduced pressure. A recrystallisation in chloroform/ethanol $(1: 2)$ yielded $0,75 \mathrm{~g}(61 \%)$ of the pure product.

${ }^{1} \mathrm{H}-\mathrm{NMR}\left(500 \mathrm{MHz}, \mathrm{CDCl}_{3}, \delta\right): 9,01$ (s, $1 \mathrm{H}$, aromatic); $8,78(\mathrm{~m}, 5 \mathrm{H}$, aromatic); $7,85(\mathrm{~d}, 1 \mathrm{H}$, aromatic, $J=8,30)$; $7,73(\mathrm{~d}, 2 \mathrm{H}$, aromatic, $J=6,34) ; 7,63(\mathrm{~m}, 9 \mathrm{H}$, aromatic); $6,80\left(\mathrm{dd}, 1 \mathrm{H}, \mathrm{CH}_{2}=\mathrm{CHAr}, J=10,99, J=17,58\right) ; 5,85(\mathrm{~d}, 1 \mathrm{H}$, $\mathrm{CH}_{2}=\mathrm{CHAr}$ trans, $\left.J=17,58\right) ; 5,32\left(\mathrm{~d}, 1 \mathrm{H}, \mathrm{CH}_{2}=\mathrm{CHAr}\right.$ cis, $J$ $=10,99$ ).

${ }^{13} \mathrm{C}-\mathrm{NMR}\left(125 \mathrm{MHz}, \mathrm{CD}_{2} \mathrm{Cl}_{3}, \delta\right): 171,65(2,-\mathrm{C}=\mathrm{N}-)$; $171,5(1,-\mathrm{C}=\mathrm{N}-)$; 141,2 (1, C4); 140,2 (1, C4); 137,0 (1, $\left.\mathrm{C}_{4}\right) ; 136,8\left(1, \mathrm{C}_{4}\right) ; 136,4\left(1,=\mathrm{CH}_{2}\right) ; 136,2\left(1, \mathrm{C}_{4}\right) ; 132,5$ (2, $=\mathrm{CH}-) ; 131,0(1,=\mathrm{CH}-) ; 129,1(1,=\mathrm{CH}-) ; 129,0(4,=\mathrm{CH}-)$; 128,7 (4, =CH-); 127,9 (1, =CH-); 127,4 (3, =CH-); 126,8 $(2,=\mathrm{CH}-) ; 114,1\left(1, \mathrm{CH}_{2}\right)$.

Elemental Analysis. calcd. for $\mathrm{C}_{29} \mathrm{H}_{21} \mathrm{~N}_{3}$ (MW 411,51g mole $^{-1}$ ): C: 84,$65 ; \mathrm{H}: 5,14 ; \mathrm{N}: 10,21$; found: C: 85,$38 ; \mathrm{H}: 5,16$; $\mathrm{N}: 10,18$.

2-(3-Bromo-Phenyl)-4,6-Bis-(4-Tert-Butyl-Phenyl)-[1,3,5]-Triazine (3a, $\mathbf{R}_{\mathbf{1}}=$ Tert.-Butyl). 1,67 g $(0,013 \mathrm{~mol})$ aluminium trichloride was exhibited. After the addition of $5,3 \mathrm{~mL}$ $(0,0314 \mathrm{~mol})$ 4-tert-butylbenzonitrile, $1,7 \mathrm{~mL}(0,0126 \mathrm{~mol})$ 3 -bromobenzoylchloride, and $0,3 \mathrm{~mL}(0,0038 \mathrm{~mol})$ thionylchloride, the chemicals were dissolved in $25 \mathrm{~mL} \mathrm{1,2-dichlo-}$ robenzene. The mixture was stirred for 20 hours at $100^{\circ} \mathrm{C}$. After 1 hour, $1,3 \mathrm{~g}(0,025 \mathrm{~mol})$ ammonium chloride was added to the reaction.

After cooling to room temperature, the reaction mixture was poured into $100 \mathrm{~mL}$ methanol and stirred for 45 minutes. The solid product was filtered and washed with hot ethanol. After drying under reduced pressure at $60^{\circ} \mathrm{C}$, we yielded $2,98 \mathrm{~g}(48 \%)$ of a solid white product.

${ }^{1} \mathrm{H}-\mathrm{NMR}\left(500 \mathrm{MHz}, \mathrm{CDCl}_{3}, \delta\right): 8,88(\mathrm{~s}, 1 \mathrm{H}$, aromatic); $8,67(\mathrm{~m}, 5 \mathrm{H}$, aromatic); 7,72 (d, $1 \mathrm{H}$, aromatic, $J=8,05) ; 7,60$ (d, $4 \mathrm{H}$, aromatic, $J=8,55) ; 7,44(\mathrm{t}, 1 \mathrm{H}$, aromatic, $J=8,05)$; $1,41\left(\mathrm{~s}, 18 \mathrm{H}, \mathrm{CH}_{3}=\mathrm{CHAr}, J=18,0\right)$.

2,4-Bis-(4-Tert-Butyl-Phenyl)-6-(4'-Vinyl-Biphenyl-3-yl)-[1,3,5]Triazine (8a). 2,0 g (0,004 mol) (3a, $\mathbf{R}_{1}=$ Tert.-Butyl), $0,89 \mathrm{~g}(0,006 \mathrm{~mol}) 4$-vinylphenylboronic acid (4) and 0,05 g $(0,04 \mathrm{mmol})$ tetrakis (triphenylphosphine)palladium $(0)$ were dissolved in $54 \mathrm{~mL}$ THF, and after the addition of $26 \mathrm{~mL}$ $1 \mathrm{M}$ sodium carbonate solution, the mixture was stirred for 24 hours at reflux. After cooling to room temperature, the mixture was filtered over celite, and ethyl acetate was added. The mixture was washed with water three times, and the solvent of the organic layers was removed under reduced pressure. A recrystallisation in chloroform/ethanol $(1: 2)$ yielded $0,85 \mathrm{~g}(41 \%)$ of the pure product.

${ }^{1} \mathrm{H}-\mathrm{NMR}\left(500 \mathrm{MHz}, \mathrm{CDCl}_{3}, \delta\right): 9,00(\mathrm{~s}, 1 \mathrm{H}$, aromatic); $8,74(\mathrm{~d}, 1 \mathrm{H}$, aromatic, $J=6,59) ; 8,70(\mathrm{~d}, 4 \mathrm{H}$, aromatic, $J=$ $4,88) ; 7,83(\mathrm{~d}, 1 \mathrm{H}$, aromatic, $J=7,56) ; 7,72(\mathrm{~d}, 2 \mathrm{H}$, aromatic, $J=7,80) ; 7,61\left(\mathrm{~m}, 7 \mathrm{H}\right.$, aromatic); 6,80 (dd, $1 \mathrm{H}, \mathrm{CH}_{2}=\mathrm{CHAr}$, $J=10,98,17,58) ; 5,84$ (d, $1 \mathrm{H}, \mathrm{CH}_{2}=\mathrm{CHAr}$, trans, $J=17,58$ ); 5,31 (d, $1 \mathrm{H}, \mathrm{CH}_{2}=\mathrm{CHAr}$, cis, $\left.J=10,98\right)$.

${ }^{13} \mathrm{C}-\mathrm{NMR}\left(125 \mathrm{MHz}, \mathrm{CD}_{2} \mathrm{Cl}_{3}, \delta\right): 171,5(2,-\mathrm{C}=\mathrm{N}-)$; $171,3(1,-\mathrm{C}=\mathrm{N}-) ; 156,0\left(2, \mathrm{C}_{4}\right) ; 141,1\left(1, \mathrm{C}_{4}\right) ; 140,2\left(1, \mathrm{C}_{4}\right)$; $137,0\left(1, \mathrm{C}_{4}\right) ; 136,9\left(1, \mathrm{CH}_{2}\right) ; 136,4\left(1,=\mathrm{CH}_{2}\right) ; 133,6\left(2, \mathrm{C}_{4}\right)$; $130,8(1,=\mathrm{CH}-) ; 129,1$ ( $1,=\mathrm{CH}-) ; 128,8(4,=\mathrm{CH}-) ; 127,8(1$, $=\mathrm{CH}-) ; 127,4(3,=\mathrm{CH}-) ; 126,8(2,=\mathrm{CH}-) ; 125,6$ (4, =CH-); $114,1\left(1,-\mathrm{CH}_{2}\right) ; 35,1\left(2,-\mathrm{C}-\mathrm{CH}_{3}\right) ; 31,2\left(6,-\mathrm{CH}_{3}\right)$. 
Elemental Analysis. calcd. for $\mathrm{C}_{37} \mathrm{H}_{37} \mathrm{~N}_{3}$ (MW 523,73 g mole $^{-1}$ ): C: 84,$86 ; \mathrm{H}: 7,12 \mathrm{~N}: 8,02$; found: C: 84,$94 ; \mathrm{H}: 7,34$; $\mathrm{N}: 8,02$.

2-(3-Bromo-Phenyl)-4,6-Bis-(4-Ethyl-Phenyl)-[1,3,5]-Triazine (3a, $\mathbf{R}_{\mathbf{1}}=$ Ethyl). 1,21 g $(0,018 \mathrm{~mol})$ aluminium trichloride was exhibited. After the addition of $2,6 \mathrm{~mL}(0,038 \mathrm{~mol})$ 4-ethylbenzonitrile, $1,2 \mathrm{~mL}(0,018 \mathrm{~mol})$ 3-bromobenzoylchloride, and $0,2 \mathrm{~mL}(0,0054 \mathrm{~mol})$ thionylchloride, the chemicals were dissolved in $40 \mathrm{~mL} \mathrm{1,2-dichlorobenzene.} \mathrm{The}$ mixture was stirred for 20 hours at $100^{\circ} \mathrm{C}$. After 1 hour, $0,79 \mathrm{~g}(0,036 \mathrm{~mol})$ ammonium chloride was added to the reaction. After cooling to room temperature, the reaction mixture was poured into $10 \mathrm{~mL}$ methanol and stirred for 45 minutes. The solid product was filtered and washed with hot ethanol. After drying under reduced pressure at $60^{\circ} \mathrm{C}$, we yielded $1,79 \mathrm{~g}(45 \%)$ of a solid white product.

${ }^{1} \mathrm{H}-\mathrm{NMR}\left(500 \mathrm{MHz}, \mathrm{CDCl}_{3}, \delta\right): 8,88$ (s, $1 \mathrm{H}$, aromatic); $8,69(\mathrm{~d}, 1 \mathrm{H}$, aromatic, $J=7,82) ; 8,66(\mathrm{~d}, 4 \mathrm{H}$, aromatic, $J=$ $5,13) ; 7,72(\mathrm{~d}, 1 \mathrm{H}$, aromatic, $J=7,81) ; 7,44(\mathrm{t}, 1 \mathrm{H}$, aromatic); $7,41(\mathrm{~d}, 4 \mathrm{H}$, aromatic, $J=8,06) ; 2,78\left(\mathrm{q}, 4 \mathrm{H}, \mathrm{CH}_{2}=\mathrm{CH}_{3}, J=\right.$ $7,57) ; 1,32\left(\mathrm{t}, 6 \mathrm{H}, \mathrm{CH}_{3}=\mathrm{CH}_{2}\right)$.

2,4-Bis-(4-Ethyl-Phenyl)-6-(4' - Vinyl-Biphenyl-3-yl)-[1,3,5]Triazine (7a). 1,7 g (0,0038 mol) (3a, $\mathbf{R}_{1}=$ Ethyl), 0,94 g $(0,0064 \mathrm{~mol})$ 4-vinylphenylboronic acid (4) and $0,04 \mathrm{~g}$ $(0,026 \mathrm{mmol}) \quad$ tetrakis(triphenylphosphine)palladium $(0)$ were dissolved in $65 \mathrm{~mL}$ THF, and after the addition of $38 \mathrm{~mL}$ $1 \mathrm{M}$ sodium carbonate solution, the mixture was stirred for 24 hours at reflux. After cooling to room temperature, the mixture was filtered over celite, and ethyl acetate was added. The mixture was washed with water three times, and the solvent of the organic layers was removed under reduced pressure. A recrystallisation in chloroform/ethanol $(1: 2)$ and a column chromatography in hexane/toluene $(4: 1)$ yielded $0,52 \mathrm{~g}(29 \%)$ of the pure product.

${ }^{1} \mathrm{H}-\mathrm{NMR}\left(500 \mathrm{MHz}, \mathrm{CDCl}_{3}, \delta\right.$ ): 8,98 (s, $1 \mathrm{H}$, aromatic); $8,74(\mathrm{~d}, 1 \mathrm{H}$, aromatic, $J=7,80) ; 8,69(\mathrm{~d}, 1 \mathrm{H}$, aromatic, $J=$ $6,59) ; 7,82(\mathrm{~d}, 1 \mathrm{H}$, aromatic, $J=6,35) ; 7,72(\mathrm{~d}, 2 \mathrm{H}$, aromatic, $J=6,59) ; 7,63(\mathrm{t}, 1 \mathrm{H}$, aromatic, $J=7,57) ; 7,57(\mathrm{~d}, 2 \mathrm{H}$, aromatic, $J=8,30) ; 7,40(\mathrm{~d}, 4 \mathrm{H}$, aromatic, $J=8,06) ; 6,79$ (dd, $\left.1 \mathrm{H}, \mathrm{CH}_{2}=\mathrm{CHAr}, J=10,98, J=17,57\right) ; 5,84(\mathrm{~d}, 1 \mathrm{H}$, $\mathrm{CH}_{2}=\mathrm{CHAr}$, trans, $\left.J=17,57\right) ; 5,31\left(\mathrm{~d}, 1 \mathrm{H}, \mathrm{CH}_{2}=\mathrm{CHAr}\right.$, cis, $J=10,98) ; 2,78$ (q, 2H, $\left.\mathrm{CH}_{2}, J=7,65\right) ; 1,32\left(\mathrm{t}, 3 \mathrm{H}, \mathrm{CH}_{3}, J=\right.$ 7,57).

${ }^{13} \mathrm{C}-\mathrm{NMR}\left(125 \mathrm{MHz}, \mathrm{CD}_{2} \mathrm{Cl}_{3}, \delta\right): 171,5(2,-\mathrm{C}=\mathrm{N}-)$; $171,3(1,-\mathrm{C}=\mathrm{N}-) ; 149,3\left(2, \mathrm{C}_{4}\right) ; 141,1\left(1, \mathrm{C}_{4}\right) ; 140,2\left(1, \mathrm{C}_{4}\right)$; $137,0\left(1, \mathrm{C}_{4}\right) ; 136,9\left(1, \mathrm{CH}_{2}\right) ; 136,4\left(1,=\mathrm{CH}_{2}\right) ; 133,8\left(2, \mathrm{C}_{4}\right)$; 130,8 (1, =CH-); 129,1 (4, =CH-); 129,0 (1, =CH-); 128,2 (4, $=\mathrm{CH}-) ; 127,8$ (1, =CH-); 127,4 (2, =CH-); 127,4 (2, =CH-); 126,7 (1, =CH-); 114,1 (1, $\left.-\mathrm{CH}_{2}\right) ; 29,0\left(2,-\mathrm{CH}_{2}-\right) ; 15,4$ (2, $\left.-\mathrm{CH}_{3}\right)$.

Elemental Analysis. calcd. for $\mathrm{C}_{33} \mathrm{H}_{29} \mathrm{~N}_{3}$ (MW 467,62 g mole $\left.{ }^{-1}\right)$ : C: 84,76 ; H: 6,25 N: 8,99; found: C: 84,$36 ; \mathrm{H}: 6,16$; $\mathrm{N}: 8,91$.
2-(3-Bromo-Phenyl)-4,6-di-p-Tolyl-[1,3,5]-Triazine $\left(\mathbf{3} \mathbf{a}, \mathbf{R}_{\mathbf{1}}=\right.$ Methyl). 1,63 g (0,012 mol) aluminium trichloride and 3,0 $\mathrm{g}$ $(0,026 \mathrm{~mol}) \mathrm{p}$-tolunitrile were exhibited. After the addition of 1,6 mL (0,012 mol) 3-bromobenzoylchloride and 0,3 mL $(0,0037 \mathrm{~mol})$ thionylchloride, the chemicals were dissolved in $100 \mathrm{~mL} \mathrm{1,2-dichlorobenzene.} \mathrm{The} \mathrm{mixture} \mathrm{was} \mathrm{stirred}$ for 20 hours at $140^{\circ} \mathrm{C}$. After 1 hour, $1,31 \mathrm{~g}(0,0244 \mathrm{~mol})$ ammonium chloride was added to the reaction. After cooling to room temperature, the solvent was completely removed, and the product was isolated by column chromatography in hexane/toluene $(8: 1)$, which yielded $1,23 \mathrm{~g}(24 \%)$ of a solid white product.

${ }^{1} \mathrm{H}-\mathrm{NMR}\left(500 \mathrm{MHz}, \mathrm{CDCl}_{3}, \delta\right): 8,87$ (s, $1 \mathrm{H}$, aromatic); $8,69(\mathrm{~d}, 1 \mathrm{H}$, aromatic, $J=7,81) ; 8,64(\mathrm{~d}, 4 \mathrm{H}$, aromatic, $J=$ $6,59) ; 7,72(\mathrm{~d}, 1 \mathrm{H}$, aromatic, $J=8,79) ; 7,44(\mathrm{t}, 1 \mathrm{H}$, aromatic, $J=7,81) ; 7,37$ (d, $4 \mathrm{H}$, aromatic, $J=8,06)$.

2,4-Di-p-Tolyl-6-(4'-Vinyl-Biphenyl-3-yl)-[1,3,5]-Triazine (6a). $1,0 \mathrm{~g}(0,0025 \mathrm{~mol})\left(3 \mathrm{a}, \mathbf{R}_{1}=\right.$ Methyl $), 0,55 \mathrm{~g}(0,0037 \mathrm{~mol})$ 4-vinylphenylboronic (4) acid and 0,02 g (0,017 mmol) tetrakis(triphenylphosphine)palladium $(0)$ were dissolved in $50 \mathrm{~mL}$ THF, and after the addition of $25 \mathrm{~mL} 1 \mathrm{M}$ sodium carbonate solution, the mixture was stirred for 24 hours at reflux. After cooling to room temperature, the mixture was filtered over celite, and ethyl acetate was added. The mixture was washed with water three times, and the solvent of the organic layers was removed under reduced pressure. Column chromatography with hexane/toluene (4:1) gave $0,73 \mathrm{~g}(67 \%)$ of the pure white powder product.

${ }^{1} \mathrm{H}-\mathrm{NMR}\left(500 \mathrm{MHz}, \mathrm{CDCl}_{3}, \delta\right): 9,04$ (s, 1H, aromatic); $8,76(\mathrm{~d}, 1 \mathrm{H}$, aromatic, $J=7,81) ; 8,70(\mathrm{~d}, 4 \mathrm{H}$, aromatic, $J=$ $8,30) ; 7,89(\mathrm{~d}, 1 \mathrm{H}$, aromatic, $J=8,30) ; 7,75(\mathrm{~d}, 2 \mathrm{H}$, aromatic, $J=6,59) ; 7,65(\mathrm{t}, 1 \mathrm{H}$, aromatic, $J=7,81) ; 7,58(\mathrm{~d}, 2 \mathrm{H}$, aromatic, $J=8,31) ; 7,39(\mathrm{~d}, 4 \mathrm{H}$, aromatic, $J=8,06) ; 6,79$ (dd, $\left.1 \mathrm{H}, \mathrm{CH}_{2}=\mathrm{CHAr}, J=10,98, J=17,82\right) ; 5,86(\mathrm{~d}, 1 \mathrm{H}$, $\mathrm{CH}_{2}=\mathrm{CHAr}$, trans, $\left.J=17,82\right) ; 5,26\left(\mathrm{~d}, 1 \mathrm{H}, \mathrm{CH}_{2}=\mathrm{CHAr}\right.$, cis, $J=10,98) ; 2,46\left(\mathrm{~s}, 6 \mathrm{H}, \mathrm{CH}_{2}=\mathrm{CH}_{3}\right)$.

Elemental Analysis. calcd. for $\mathrm{C}_{31} \mathrm{H}_{25} \mathrm{~N}_{3}$ (MW 439,57 g mole ${ }^{-1}$ ): C: 84,71 ; H: $5,73 \mathrm{~N}$ : 9,56; found: C: 84,72 ; H: 5,87; $\mathrm{N}: 9,32$.

2-(4-Bromo-Phenyl)-4,6-Diphenyl-[1,3,5]-Triazine (3b). $60,59 \mathrm{~g}(0,454 \mathrm{~mol})$ aluminium trichloride was exhibited. After the addition of $9,9 \mathrm{~mL}(0,136 \mathrm{~mol})$ thionylchloride, $60 \mathrm{~mL}$ (0,454 mol) 3-bromobenzoylchloride and $98,4 \mathrm{~mL}$ benzonitrile, the chemicals were dissolved in $800 \mathrm{~mL} \mathrm{1,2-}$ dichlorobenzene. The mixture was stirred for 20 hours at $100^{\circ} \mathrm{C}$. After 1 hour, 48,61 $\mathrm{g}(0,909 \mathrm{~mol})$ ammonium chloride was added to the reaction. After cooling to room temperature, the reaction mixture was poured into $3 \mathrm{~L}$ methanol and stirred for 45 minutes. The solid product was filtered and washed with hot ethanol. After drying under reduced pressure at $60^{\circ} \mathrm{C}$, we yielded $36,69 \mathrm{~g}(20 \%)$ of a solid white product.

${ }^{1} \mathrm{H}-\mathrm{NMR}\left(500 \mathrm{MHz}, \mathrm{CDCl}_{3}, \delta\right): 8,77$ (d, 4H, aromatic, $J=6,84) ; 8,65(\mathrm{~d}, 2 \mathrm{H}$, aromatic, $J=8,06) ; 7,71(\mathrm{~d}, 2 \mathrm{H}$, aromatic, $J=8,78) ; 7,60$ (m, $6 \mathrm{H}$, aromatic). 
2,4-Diphenyl-6-(4'-Vinyl-Biphenyl-4-yl)-[1,3,5]-Triazine (5b). $1,16 \mathrm{~g} \quad(0,003 \mathrm{~mol}) \quad(3 \mathbf{b}), \quad 0,74 \mathrm{~g} \quad(0,005 \mathrm{~mol}) \quad 4$-vinylphenylboronic acid (4) and $0,02 \mathrm{~g}(0,02 \mathrm{mmol})$ tetrakis (triphenylphosphine)palladium(0) were dissolved in $45 \mathrm{~mL}$ THF, and after the addition of $30 \mathrm{~mL} 1 \mathrm{M}$ sodium carbonate solution, the mixture was stirred for 24 hours at reflux. After cooling to room temperature, the mixture was filtered over celite, and ethyl acetate was added. The mixture was washed with water three times, and the solvent of the organic layers was removed under reduced pressure. A recrystallisation in chloroform/ethanol $(1: 2)$ yielded $0,75 \mathrm{~g}(61 \%)$ of the pure product.

${ }^{1} \mathrm{H}-\mathrm{NMR}\left(500 \mathrm{MHz}, \mathrm{CDCl}_{3}, \delta\right): 8,85(\mathrm{~d}, 2 \mathrm{H}$, aromatic, $J=8,54) ; 8,80(\mathrm{~d}, 4 \mathrm{H}$, aromatic, $J=4,89) ; 7,82(\mathrm{~d}, 2 \mathrm{H}$, aromatic, $J=8,44) ; 7,69(\mathrm{~d}, 2 \mathrm{H}$, aromatic, $J=8,30) ; 7,61$ (m, $6 \mathrm{H}$, aromatic); 7,55 (d, $2 \mathrm{H}$, aromatic, $J=8,30) ; 6,78$ (dd, $\left.1 \mathrm{H}, \mathrm{CH}_{2}=\mathrm{CHAr}, J=10,98,17,58\right) ; 5,69\left(\mathrm{~d}, 1 \mathrm{H}, \mathrm{CH}_{2}=\mathrm{CHAr}\right.$, trans, $J=17,58) ; 5,32\left(\mathrm{~d}, 1 \mathrm{H}, \mathrm{CH}_{2}=\mathrm{CHAr}\right.$, cis, $\left.J=10,98\right)$.

${ }^{13} \mathrm{C}-\mathrm{NMR}\left(125 \mathrm{MHz}, \mathrm{CD}_{2} \mathrm{Cl}_{3}, \delta\right): 171,6(2,-\mathrm{C}=\mathrm{N}-)$; $171,3(1,-\mathrm{C}=\mathrm{N}-) ; 144,6\left(1, \mathrm{C}_{4}\right) ; 139,6\left(1, \mathrm{C}_{4}\right) ; 137,3(1$, $\left.\mathrm{C}_{4}\right) ; 136,3\left(1,=\mathrm{CH}_{2}\right) ; 136,3\left(2, \mathrm{C}_{4}\right) ; 135,2\left(1, \mathrm{C}_{4}\right) ; 132,5(2$, $\left.=\mathrm{CH}_{2}\right) ; 129,5(2,=\mathrm{CH}-) ; 129,0(4,=\mathrm{CH}-) ; 128,6(4,=\mathrm{CH}-)$; 127,4 (2, =CH-); 127,1 (2, =CH-); 126,8 (2, =CH-); 114,1 $\left(1, \mathrm{CH}_{2}\right)$.

Elemental Analysis. calcd. for $\mathrm{C}_{29} \mathrm{H}_{21} \mathrm{~N}_{3}$ (MW 411,51g mole $^{-1}$ ): C: 84,$65 ; \mathrm{H}: 5,14 \mathrm{~N}: 10,21$; found: C: 84,$56 ; \mathrm{H}: 5,16$; N: 9,84 .

2-(4-Bromo-Phenyl)-4,6-Bis-(4-Tert-Butyl-Phenyl)-[1,3,5]-Triazine (3b, $\mathbf{R}_{\mathbf{1}}=$ Tert.-Butyl). 1,67 $\mathrm{g}(0,013 \mathrm{~mol})$ aluminium trichloride, 5,0 g (0,031 mol) 4-tert-butylbenzonitrile, 2,76 g $(0,126 \mathrm{~mol})$ 4-bromobenzoylchloride were exhibited. After the addition of $0,3 \mathrm{~mL}(0,0038 \mathrm{~mol})$ thionylchloride, the chemicals were dissolved in $25 \mathrm{~mL} \mathrm{1,2-dichlorobenzene.} \mathrm{The}$ mixture was stirred for 20 hours at $100^{\circ} \mathrm{C}$. After 1 hour, $1,34 \mathrm{~g}(0,025 \mathrm{~mol})$ ammonium chloride was added to the reaction. After cooling to room temperature, the reaction mixture was poured into $500 \mathrm{~mL}$ methanol and stirred for 45 minutes. The solid product was filtered and dried under reduced pressure at $60^{\circ} \mathrm{C}$. The white product powder yielded $3,10 \mathrm{~g}(21 \%)$.

${ }^{1} \mathrm{H}-\mathrm{NMR}\left(500 \mathrm{MHz}, \mathrm{THF}-\mathrm{d}_{8}, \delta\right): 8,64(\mathrm{~m}, 4 \mathrm{H}$, aromatic); $7,68(\mathrm{~d}, 2 \mathrm{H}$, aromatic, $J=8,54) ; 7,58(\mathrm{~d}, 4 \mathrm{H}$, aromatic, $J=$ $8,55) ; 1,40\left(\mathrm{~s}, 18 \mathrm{H},\left(\mathrm{CH}_{3}\right)_{2}\right)$.

2,4-Bis-(4-Tert-Butyl-Phenyl)-6-(4'-Vinyl-Biphenyl-4-yl)-[1,3,5]Triazine (8b). $2,0 \mathrm{~g} \quad(0,004 \mathrm{~mol}) \quad\left(\mathbf{3 b}, \mathbf{R}_{1}=\right.$ Tert.-Butyl), $0,89 \mathrm{~g}(0,006 \mathrm{~mol}) 4$-vinylphenylboronic acid (4) and $0,05 \mathrm{~g}$ $(0,04 \mathrm{mmol})$ tetrakis(triphenylphosphine)palladium( $(0)$ were dissolved in $54 \mathrm{~mL}$ THF, and after the addition of $26 \mathrm{~mL}$ $1 \mathrm{M}$ sodium carbonate solution, the mixture was stirred for 24 hours at reflux. After cooling to room temperature, the mixture was filtered over celite, and ethyl acetate was added. The mixture was washed with water three times, and the solvent of the organic layers was removed under reduced pressure. A recrystallisation in chloroform/ethyl acetate yielded $1,60 \mathrm{~g}(76 \%)$ of the pure product.
${ }^{1} \mathrm{H}-\mathrm{NMR}\left(500 \mathrm{MHz}, \mathrm{CDCl}_{3}, \delta\right): 8,82(\mathrm{~d}, 2 \mathrm{H}$, aromatic, $J=8,30) ; 8,69(\mathrm{~d}, 4 \mathrm{H}$, aromatic, $J=8,31) ; 7,79(\mathrm{~d}, 2 \mathrm{H}$, aromatic, $J=8,30) ; 7,67(\mathrm{~d}, 2 \mathrm{H}$, aromatic, $J=8,30) ; 7,60(\mathrm{~d}$, $4 \mathrm{H}$, aromatic, $J=8,30) ; 6,78\left(\mathrm{dd}, 1 \mathrm{H}, \mathrm{CH}_{2}=\mathrm{CHAr}, J=10,98\right.$, $17,58) ; 5,83\left(\mathrm{~d}, 1 \mathrm{H}, \mathrm{CH}_{2}=\mathrm{CHAr}\right.$, trans, $\left.J=17,58\right) ; 5,31(\mathrm{~d}$, $1 \mathrm{H}, \mathrm{CH}_{2}=\mathrm{CHAr}$, cis, $J=10,99$ ).

${ }^{13} \mathrm{C}-\mathrm{NMR}\left(125 \mathrm{Mhz}, \mathrm{CDCl}_{3}, \delta\right): 171,5(2,-\mathrm{C}=\mathrm{N}-) ; 171,1$ $(1,-\mathrm{C}=\mathrm{N}-) ; 156,0\left(2, \mathrm{C}_{4}\right) ; 144,4\left(1, \mathrm{C}_{4}\right) ; 139,8\left(1, \mathrm{C}_{4}\right) ; 137,3$ $\left(1, \mathrm{C}_{4}\right) ; 136,3\left(1, \mathrm{CH}_{2}\right) ; 135,4\left(1, \mathrm{C}_{4}\right) ; 133,7\left(2, \mathrm{C}_{4}\right) ; 129,4(2$, $=\mathrm{CH}-) ; 128,8(4,=\mathrm{CH}-) ; 127,4(2,=\mathrm{CH}-) ; 127,0(2,=\mathrm{CH}-)$; $126,8(2,=\mathrm{CH}-) ; 125,6(4,=\mathrm{CH}-) ; 114,3\left(1,=\mathrm{CH}_{2}\right) ; 35,1(2$, $\left.-\mathrm{C}-\mathrm{CH}_{3}\right) ; 31,2\left(6,-\mathrm{CH}_{3}\right)$.

Elemental Analysis. calcd. for $\mathrm{C}_{37} \mathrm{H}_{37} \mathrm{~N}_{3}$ (MW 523,73 g mole $^{-1}$ ): C: 84,$86 ; \mathrm{H}: 7,12 \mathrm{~N}: 8,02$; found: C: 86,13 ; H: 7,34; $\mathrm{N}: 7,38$.

2-(4-Bromo-Phenyl)-4,6-di-p-Tolyl-[1,3,5]-Triazine $\left(\mathbf{3} \mathbf{b}, \mathbf{R}_{\mathbf{1}}=\right.$ Methyl). 1,2 g (0,009 mol) aluminium trichloride, $1,99 \mathrm{~g}$ $(0,009 \mathrm{~mol})$ 4-bromobenzoylchloride were exhibited. After the addition of $2,3 \mathrm{~mL}(0,02 \mathrm{~mol}) \mathrm{p}$-tolunitrile and $0,2 \mathrm{~mL}$ $(0,0027 \mathrm{~mol})$ thionylchloride, the chemicals were dissolved in $25 \mathrm{~mL} \mathrm{1,2-dichlorobenzene.} \mathrm{The} \mathrm{mixture} \mathrm{was} \mathrm{stirred} \mathrm{for} 20$ hours at $130^{\circ} \mathrm{C}$. After 1 hour, $0,97 \mathrm{~g}(0,018 \mathrm{~mol})$ ammonium chloride was added to the reaction. After cooling to room temperature, the reaction mixture was poured into $20 \mathrm{~mL}$ methanol and stirred for 45 minutes. The solid product was filtered and dried under reduced pressure at $60^{\circ} \mathrm{C}$. The white product powder yielded $1,10 \mathrm{~g}(29 \%)$.

${ }^{1} \mathrm{H}-\mathrm{NMR}\left(500 \mathrm{MHz}, \mathrm{CDCl}_{3}, \delta\right): 8,62(\mathrm{~m}, 6 \mathrm{H}$, aromatic); $7,69(\mathrm{~d}, 2 \mathrm{H}$, aromatic, $J=8,54) ; 7,37(\mathrm{~d}, 4 \mathrm{H}$, aromatic, $J=$ $8,06) ; 2,48\left(\mathrm{~s}, 6 \mathrm{H}, \mathrm{CH}_{2}=\mathrm{CH}_{3}\right.$ ).

2,4-Di-p-Tolyl-6-(4'-Vinyl-Biphenyl-4-yl)-[1,3,5]-Triazine (6b). $1,0 \mathrm{~g}(0,0024 \mathrm{~mol}) \quad\left(3 \mathbf{b}, \mathbf{R}_{1}=\right.$ Methyl $), 0,59 \mathrm{~g}(0,004 \mathrm{~mol})$ 4-vinylphenylboronic acid (4) and $0,02 \mathrm{~g}(0,02 \mathrm{mmol})$ tetrakis(triphenylphosphine)palladium $(0)$ were dissolved in $50 \mathrm{~mL}$ THF, and after the addition of $24 \mathrm{~mL} 1 \mathrm{M}$ sodium carbonate solution, the mixture was stirred for 24 hours at reflux. After cooling to room temperature, the mixture was filtered over celite, and ethyl acetate was added. The mixture was washed with water three times, and the solvent of the organic layers was removed under reduced pressure. A column chromatography in hexane/toluene $(8: 1)$ yielded $0,41 \mathrm{~g}(39 \%)$ of the pure white product.

${ }^{1} \mathrm{H}-\mathrm{NMR}\left(500 \mathrm{MHz}, \mathrm{CDCl}_{3}, \delta\right): 8,83(\mathrm{~d}, 2 \mathrm{H}$, aromatic, $J=8,54) ; 8,68(\mathrm{~d}, 4 \mathrm{H}$, aromatic, $J=8,06) ; 7,81(\mathrm{~d}, 2 \mathrm{H}$, aromatic, $J=8,30) ; 7,69(\mathrm{~d}, 2 \mathrm{H}$, aromatic, $J=8,05) ; 7,55$ $(\mathrm{d}, 2 \mathrm{H}$, aromatic, $J=8,30), 7,38(\mathrm{~d}, 4 \mathrm{H}$, aromatic, $J=8,06)$; $6,78\left(\mathrm{dd}, 1 \mathrm{H}, \mathrm{CH}_{2}=\mathrm{CHAr}, J=10,98,17,58\right) ; 5,84(\mathrm{~d}, 1 \mathrm{H}$, $\mathrm{CH}_{2}=\mathrm{CHAr}$, trans, $\left.J=17,58\right) ; 5,32\left(\mathrm{~d}, 1 \mathrm{H}, \mathrm{CH}_{2}=\mathrm{CHAr}\right.$, cis, $J=10,99) ; 2,49\left(\mathrm{~s}, 6 \mathrm{H}, \mathrm{CH}_{2}=\mathrm{CH}_{3}\right)$.

${ }^{13} \mathrm{C}-\mathrm{NMR}\left(125 \mathrm{MHz}, \mathrm{CD}_{2} \mathrm{Cl}_{3}, \delta\right): 171,5(2,-\mathrm{C}=\mathrm{N}-)$; $171,1(1,-\mathrm{C}=\mathrm{N}-) ; 144,4\left(1, \mathrm{C}_{4}\right) ; 143,0\left(2, \mathrm{C}_{4}\right) ; 139,7\left(1, \mathrm{C}_{4}\right)$; $137,3\left(1, \mathrm{C}_{4}\right) ; 136,3\left(1,=\mathrm{CH}_{2}\right) ; 135,4\left(1, \mathrm{C}_{4}\right) ; 133,7\left(2, \mathrm{C}_{4}\right)$; $129,4(2,=\mathrm{CH}-) ; 129,4(4,=\mathrm{CH}-) ; 128,9(4,=\mathrm{CH}-) ; 127,4(2$, $=\mathrm{CH}-) ; 127,0(2,=\mathrm{CH}-) ; 126,8(2,=\mathrm{CH}-) ; 114,3\left(1,-\mathrm{CH}_{2}\right)$; $21,8\left(2,-\mathrm{CH}_{3}\right)$. 
Elemental Analysis. calcd. for $\mathrm{C}_{31} \mathrm{H}_{25} \mathrm{~N}_{3}$ (MW 439,57g mole $\left.{ }^{-1}\right)$ : C: 84,$71 ; \mathrm{H}: 5,73 \mathrm{~N}: 9,56$; found: C: 85,10 ; H: 5,84; $\mathrm{N}: 9,48$.

2-(4-Bromo-Phenyl)-4,6-Bis-(4-Ethyl-Phenyl)-[1,3,5]-Triazine (3b, $\mathbf{R}_{\mathbf{1}}=$ Ethyl). 1,2 g (0,009 mol) aluminium trichloride and $1,99 \mathrm{~g} \quad(0,009 \mathrm{~mol})$ 4-bromobenzoylchloride were exhibited. After the addition of $2,3 \mathrm{~mL}(0,02 \mathrm{~mol}) 4$ ethylbenzonitrile and $0,2 \mathrm{~mL}(0,0027 \mathrm{~mol})$ thionylchloride, the chemicals were dissolved in $40 \mathrm{~mL} \mathrm{1,2-dichlorobenzene.}$ The mixture was stirred for 20 hours at $130^{\circ} \mathrm{C}$. After 1 hour, $0,97 \mathrm{~g}(0,018 \mathrm{~mol})$ ammonium chloride was added to the reaction. After cooling to room temperature, the reaction mixture was poured into $20 \mathrm{~mL}$ methanol and stirred for 45 minutes. The solid product was filtered, washed with hot ethanol and dried under reduced pressure at $60^{\circ} \mathrm{C}$. The white product powder yielded $0,87 \mathrm{~g}(22 \%)$.

${ }^{1} \mathrm{H}-\mathrm{NMR}\left(500 \mathrm{MHz} \mathrm{CDCl}_{3}, \delta\right): 8,65$ (m, 6H, aromatic); $7,70(\mathrm{~d}, 2 \mathrm{H}$, aromatic, $J=8,79) ; 7,40(\mathrm{~d}, 4 \mathrm{H}$, aromatic, $J=8,55) ; 2,78\left(\mathrm{q}, 4 \mathrm{H}, \mathrm{CH}_{2}=\mathrm{CH}_{3}, J=7,56\right) ; 1,32(\mathrm{t}, 6 \mathrm{H}$, $\left.\mathrm{CH}_{3}=\mathrm{CH}_{2}, J=7,56\right)$.

2,4-Bis-(4-Ethyl-Phenyl)-6-(4' -Vinyl-Biphenyl-4-yl)-[1,3,5]Triazine (7b). $0,8 \mathrm{~g}(0,0018 \mathrm{~mol}) \quad\left(3 \mathbf{b}, \mathbf{R}_{1}=\right.$ Ethyl $), 0,4 \mathrm{~g}$ $(0,0027 \mathrm{~mol})$ 4-vinylphenylboronic acid (4) and $0,01 \mathrm{~g}$ $(0,012 \mathrm{mmol}) \quad$ tetrakis(triphenylphosphine)palladium $(0)$ were dissolved in $40 \mathrm{~mL}$ THF, and after the addition of $18 \mathrm{~mL}$ $1 \mathrm{M}$ sodium carbonate solution, the mixture was stirred for 24 hours at reflux. After cooling to room temperature, the mixture was filtered over celite, and ethyl acetate was added. The mixture was washed with water three times, and the solvent of the organic layers was removed under reduced pressure. A column chromatography in hexane/toluene $(4: 1)$ yielded $0,5 \mathrm{~g}(59 \%)$ of the pure white product.

${ }^{1} \mathrm{H}-\mathrm{NMR}\left(500 \mathrm{MHz}, \mathrm{CDCl}_{3}, \delta\right): 8,81$ (d, 2H, aromatic, $J=8,54) ; 8,69(\mathrm{~d}, 4 \mathrm{H}$, aromatic, $J=8,30) ; 7,78(\mathrm{~d}, 2 \mathrm{H}$, aromatic, $J=8,30) ; 7,67(\mathrm{~d}, 2 \mathrm{H}$, aromatic, $J=8,05) ; 7,52$ $(\mathrm{d}, 2 \mathrm{H}$, aromatic, $J=8,30), 7,39(\mathrm{~d}, 4 \mathrm{H}$, aromatic, $J=8,06)$; $6,78\left(\mathrm{dd}, 1 \mathrm{H}, \mathrm{CH}_{2}=\mathrm{CHAr}, J=10,75,17,58\right) ; 5,82(\mathrm{~d}, 1 \mathrm{H}$, $\mathrm{CH}_{2}=\mathrm{CHAr}$, trans, $\left.J=17,58\right) ; 5,30\left(\mathrm{~d}, 1 \mathrm{H}, \mathrm{CH}_{2}=\mathrm{CHAr}\right.$, cis, $J=10,74) ; 2,77$ (q, 4H, $\left.\mathrm{CH}_{2}=\mathrm{CH}_{3}, J=7,57\right) ; 1,31(\mathrm{t}, 6 \mathrm{H}, J=$ $7,81)$.

${ }^{13} \mathrm{C}-\mathrm{NMR}\left(125 \mathrm{MHz}, \mathrm{CD}_{2} \mathrm{Cl}_{3}, \delta\right): 171,4(2,-\mathrm{C}=\mathrm{N}-)$; $171,0(1,-\mathrm{C}=\mathrm{N}-) ; 149,2\left(2, \mathrm{C}_{4}\right) ; 144,3\left(1, \mathrm{C}_{4}\right) ; 139,7\left(1, \mathrm{C}_{4}\right)$; $137,2\left(1, \mathrm{C}_{4}\right) ; 136,3\left(1,=\mathrm{CH}_{2}\right) ; 13,4\left(1, \mathrm{C}_{4}\right) ; 133,9\left(2, \mathrm{C}_{4}\right)$; 129,4 (2, =CH-); 129,0 (4, =CH-); 128,1 (4, =CH-); 127,3 (2, $=\mathrm{CH}-) ; 127,0$ (2, =CH-); 126,7 (2, =CH-); 114,3 (1, $\left.-\mathrm{CH}_{2}\right)$; $29,0\left(2,-\mathrm{CH}_{2}-\right) ; 15,4\left(2,-\mathrm{CH}_{3}\right)$.

Elemental Analysis. Calcd. for $\mathrm{C}_{33} \mathrm{H}_{29} \mathrm{~N}_{3}$ (MW 467,26 g mole ${ }^{-1}$ ): C: 84,76 ; H: 6,25 N: 8,99; found: C: 84,$97 ; \mathrm{H}: 6,54$; $\mathrm{N}: 8,85$.

2.1.8. General Polymerization Procedure. The polymerizations were carried out in a glove box under an argon atmosphere. For the free radical polymerization, N,Nazobisisobutyronitrile in a concentration of $2 \mathrm{~mol} \%$ was used. The monomers and the initiator were dissolved in

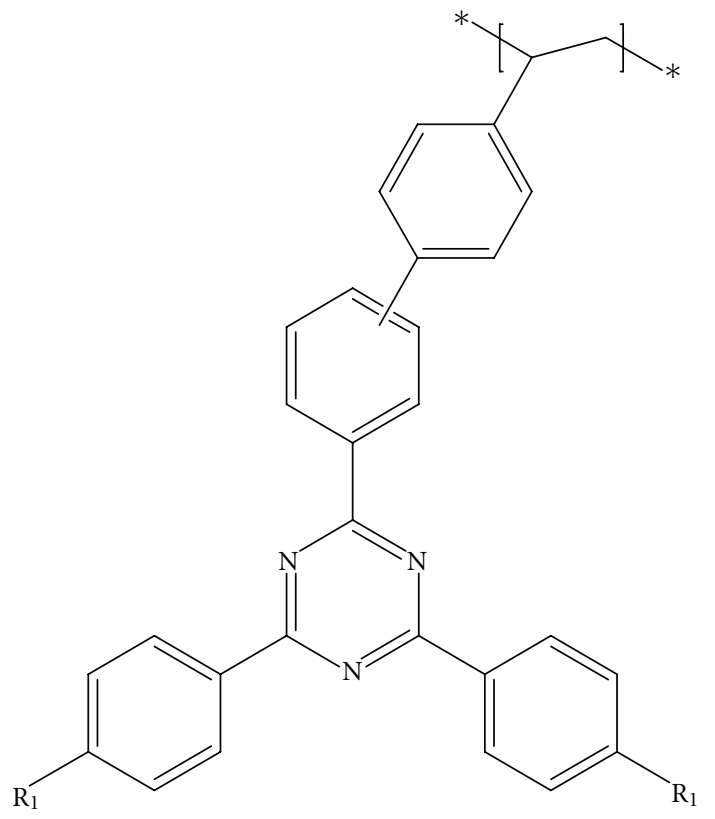

$\mathrm{R}_{1}=\mathrm{H}$; methyl; ethyl; tert-butyl groups

FIGURE 1: General structure of the electron-transporting polystyrenes $\mathbf{9 a} / \mathbf{b}-\mathbf{1 2} \mathbf{a} / \mathbf{b}$ with 2,4,6-triphenyl-1,3,5-triazine side groups (a: meta; b: para).

freshly distilled tetrahydrofuran. The solution was stirred for 72 hours at $50^{\circ} \mathrm{C}$. For purification, the polymers $\mathbf{9 a} / \mathbf{b}-\mathbf{1 2 a} / \mathbf{b}$ were diluted in THF, precipitated in methanol/diethyl ether several times, and dried under reduced pressure at $60^{\circ} \mathrm{C}$.

\section{Results and Discussion}

3.1. Synthesis of Monomers and Polymers. As a general structure for the electron-transporting polymers $\mathbf{9 a} / \mathbf{b}-\mathbf{1 2} \mathbf{a} / \mathbf{b}$, we used a polystyrene with 2,4,6-triphenyl-1,3,5-triazine side groups as it is shown in Figure 1.

The styryl group is linked to the 2,4,6-triphenyl-1,3,5triazine in two different ways-in meta- and paraposition. Furthermore, the 4- and 6-phenyl rings are substituted in paraposition by different alkyl groups $\mathrm{R} 1 \mathrm{H}$, methyl, ethyl and tert.-butyl). The aim of this work is to study the influence of these structure modifications on the solubility, the film forming properties, thermal properties and on OLED performance.

A typical phosphorescent green emitter 13 was used in combination with a small molecule cohost $\mathrm{CoH}-001$; both were delivered from Merck KGaA, Darmstadt and used as received.

In order to synthesise the triazine core structure shown in Figure 1, we used the already known nitrile route, which is described by Behl and Zentel [15]. A general scheme for the nitrile route is shown in Figure 2. 


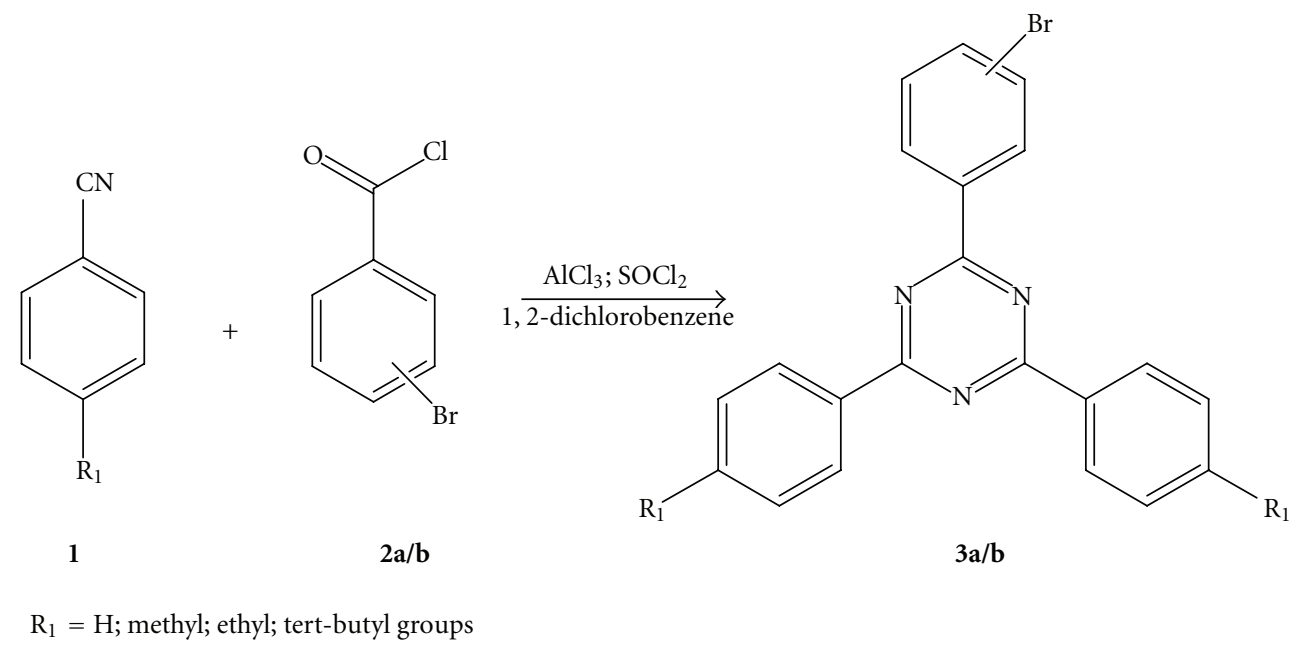

FiguRE 2: Nitrile route for building up the triazine core (a: 3-bromo-, b: 4-bromo-derivate).

The first step is the triazine formation which is done via a nitrile group exchange catalyzed by aluminium trichloride. The reactants are a bromosubstituted benzoic acid chloride and a nitrile with the appropriate side groups. As benzoic acid we used the 3-bromo (2a) and the 4-bromo derivative (2b) in order to get the different linkage for the styryl substitution in meta- or paraposition during the second step. For the building of the triazine core, the yields are often about $50 \%$ depending on the nitrile substituent used.

The second step is a Suzuki coupling of the triazine bromide (3a/b) with 4-vinylphenylboronic acid (4). This reaction step is shown in Figure 3. For this reaction, yields of up to $80 \%$ were reached.

Table 1 shows the used nitriles and the yields for the twostep monomer synthesis.

The polymerizations of the synthesized monomers were done via a free radical mechanism with AIBN as initiator under an inert atmosphere. The general polymer structure is shown in Figure 1. The monomer was dissolved in freshly distilled tetrahydrofuran (THF) and stirred at $60^{\circ} \mathrm{C}$ for three days. Afterwards, multiple precipitations in methanol/diethyl ether were done to separate monomer residue, low molecular fractions, and other impurities. After purification, the yields were about $80 \%$.

Table 2 shows the yields of the synthesized polymers to the corresponding monomers from Table 1 as well as selected analytical data for the polymers.

The molecular weights and their distribution of the polymers were characterized by GPC measurement. DSC measurements were performed to determine the thermal behavior and the glass transition for which the data is listed in Table 2. All polymers show a good solubility in toluene and chlorinated solvents such as 1,2-dichlorobenzene. For device preparation, it was possible to spin-coat good films from toluene.

In regard to the analytical data, there is an influence on the molecular weight and the glass transition temperature depending on the inserted substituent.
The weight average molecular weight lies in the range of $23 * 10^{3}$ and $100 * 10^{3} \mathrm{~g} / \mathrm{mol}$. The para tert.-butyl substituted polymer $(\mathbf{1 2 b})$ shows with $100 * 10^{3} \mathrm{~g} / \mathrm{mol}$ the highest molecular weight, while the para methyl substituted polymer (10b) shows with $23 * 10^{3} \mathrm{~g} / \mathrm{mol}$ the lowest molecular weight. The nonsubstituted structures both show an average molecular weight around $65 * 10^{3} \mathrm{~g} / \mathrm{mol}$.

The methyl and ethyl substituted polymers $\mathbf{1 0 a} / \mathbf{b}$ and 11a/b show a lower molecular weight while the tert.-butyl substituted polymers $12 \mathbf{a} / \mathbf{b}$ show a higher molecular weight in comparison to the nonsubstituted structure $\mathbf{9 a / b}$. This effect can be explained by the inserted alkyl groups for the polymers $10 \mathbf{a} / \mathbf{b}$ and $1 \mathbf{1 a} / \mathbf{b}$. Methyl or ethyl groups can alloy chain transfer with the benzyl hydrogens. Three mesomeric structures that consist of a more or less stable state can be built up. These resonance structures lower the molecular weight. As for polymer $\mathbf{1 2} \mathbf{a} / \mathbf{b}$ the tert.-butyl groups are stiff enough and do not build up mesomeric structures and therefore show a higher molecular weight.

For all polymers only glass transition temperatures $\left(T_{g}\right)$ were observed by DSC. No further thermal effects such as melting or crystallization were seen. The results for the glass transition temperature show a division between meta- and paralinked polymers. The metalinked polymers have a lower $T_{g}$ than the paralinked polymers. This effect can be explained by the stiffness of the different structures. The paralinked polymers have the ability to pack in a more compact way due to their side group while the packing of the metalinked polymers is hindered because of their twisted structure in regard to the polymer backbone. Behl and Zentel [15] found quite similar results for their vinyl-substituted 2,4,6triphenyl-1,3,5-triazine polymers compared to our styrylsubstituted analogues. The ethyl substituted polymers (11a and 11b) both show a lower $T_{g}$ in comparison to the nonsubstituted structure (9a and $\mathbf{9 b}$ ). Those flexible groups have the ability to twist within the structure and thus lower the glass transition temperature. In contrast to that, the tert.-butyl substituted polymers (12a and 12b) show 
<smiles>[R1]c1ccc(-c2nc(-c3ccc([R])cc3)nc(-c3ccc(Br)cc3)n2)cc1</smiles>

$3 \mathbf{a} / \mathbf{b}$

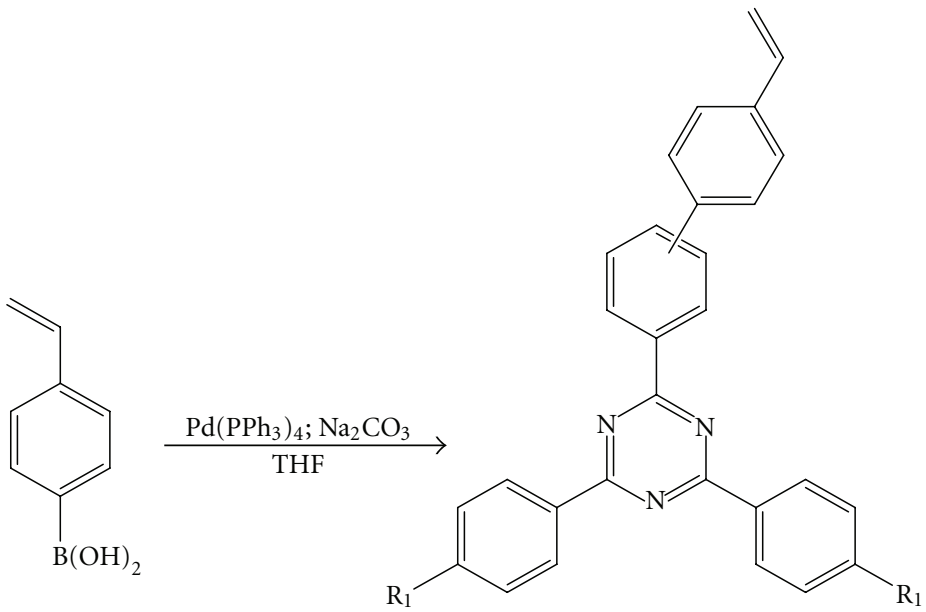

$5 a / b-8 a / b$

$\mathrm{R}_{1}=\mathrm{H}(5)$; methyl (6); ethyl (7); tert-

butyl (8) groups

FIgURE 3: Introduction of styryl functionality by Suzuki coupling (a: meta; b: para).

TABLE 1: Overview of the yields and inserted nitriles for the synthesized monomers.

\begin{tabular}{|c|c|c|c|}
\hline Number & Inserted nitrile & Inserted benzoic acid chloride & Overall yield (\%) \\
\hline $5 a$ & & 3-bromobenzoic acid chloride & 61 \\
\hline $5 b$ & & 4-bromobenzoic acid chloride & 26 \\
\hline $6 a$ & & 3-bromobenzoic acid chloride & 57 \\
\hline $6 b$ & & 4-bromobenzoic acid chloride & 67 \\
\hline $7 a$ & & 3-bromobenzoic acid chloride & 29 \\
\hline $7 \mathbf{b}$ & & 4-bromobenzoic acid chloride & 59 \\
\hline $8 \mathbf{a}$ & & 3-bromobenzoic acid chloride & 41 \\
\hline $8 b$ & & 4-bromobenzoic acid chloride & 55 \\
\hline
\end{tabular}

an increase in $T_{g}$ which can be explained by their more compact structure. A second reason could be the increasing molecular weight. For some polymers $T_{g}$ still is dependent on the polymerization degree. A polymerization degree up to 50 is accepted for a correlation between $T_{g}$, and the polymerization degree while above 50 the glass transition temperature is independent from the polymerization degree [16].

3.2. UV- and PL Spectra. Figures 4 and 5 show the UVVis spectra of the obtained polymers. In the UV-Vis spectra all polymers illustrate an absorption maximum 
TABLe 2: Analytical data of the synthesized polymers.

\begin{tabular}{lcccccccc}
\hline $\begin{array}{l}\text { Inserted } \\
\text { monomer }\end{array}$ & Polymer & \multicolumn{2}{c}{$\mathrm{GPC}\left(10^{3} \mathrm{~g} / \mathrm{mol}\right)$} & PDI & $\begin{array}{c}\text { Glass transition } \\
\text { temperature }\left({ }^{\circ} \mathrm{C}\right)\end{array}$ & Yield $(\%)$ & $\begin{array}{c}\text { UV-Vis maxima } \\
(\mathrm{nm})\end{array}$ & $\begin{array}{c}\text { PL maxima } \\
(\mathrm{nm})\end{array}$ \\
\hline $\mathbf{5 a}$ & $\mathbf{9 a}$ & 66,3 & 22,9 & 2,89 & 202 & 87 & 270 & 420 \\
$\mathbf{5 b}$ & $\mathbf{9 b}$ & 67,7 & 19,8 & 3,42 & 237 & 82 & 283 & 438 \\
$\mathbf{6 a}$ & $\mathbf{1 0 a}$ & 34,6 & 14,8 & 2,33 & 215 & 83 & 277 & 419 \\
$\mathbf{6 b}$ & $\mathbf{1 0 b}$ & 23,8 & 10,2 & 2,33 & 248 & 55 & 293 & 431 \\
$\mathbf{7 a}$ & $\mathbf{1 1 a}$ & 57,3 & 21,4 & 2,68 & 200 & 79 & 278 & 413 \\
$\mathbf{7 b}$ & $\mathbf{1 1 b}$ & 42,2 & 16,2 & 2,61 & 235 & 81 & 294 & 428 \\
$\mathbf{8 a}$ & $\mathbf{1 2 a}$ & 74,3 & 28,5 & 2,60 & 246 & 81 & 278 & 427 \\
$\mathbf{8 b}$ & $\mathbf{1 2 b}$ & 100,8 & 34,6 & 2,91 & 277 & 74 & 292 & 426 \\
\hline
\end{tabular}

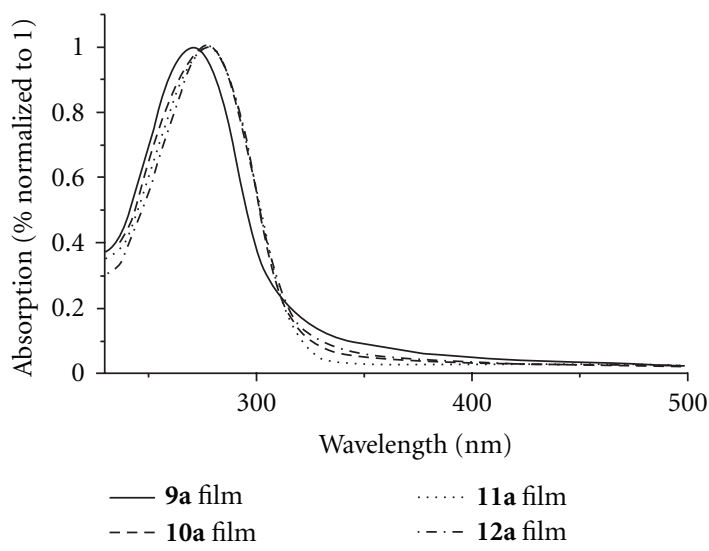

FIGURE 4: UV-spectra of metasubstituted polymer films $\mathbf{9 a - 1 2 a}$ on silica glass.

between $270 \mathrm{~nm}$ and $300 \mathrm{~nm}$. The metalinked polymers $9 \mathbf{a}-$ 12a demonstrate absorption at about $275 \mathrm{~nm}$ while the paralinked polymers $\mathbf{9 b} \mathbf{- 1 2} \mathbf{b}$ indicate absorption at about $300 \mathrm{~nm}$. The nonsubstituted metalinked polymer 9a shows the lowest absorption maximum at $270 \mathrm{~nm}$. The substituted metalinked polymers $\mathbf{1 0 a}-\mathbf{1 2} \mathbf{a}$ present shifted absorption maxima by about $7-8 \mathrm{~nm}$. The nonsubstituted paralinked polymer 9 b exhibits an absorption maximum at $283 \mathrm{~nm}$. Substituted paralinked polymers $\mathbf{1 0 b}-\mathbf{1 2}$ b show the furthest shift to higher wavelength absorption (around $10 \mathrm{~nm}$ ). This bathochromic shift can be explained by the + I-effect of the alkyl groups. For larger alkyl groups especially in the case of paralinked polymers the $+\mathrm{I}$-effect becomes stronger so that the furthest wavelength shift can be seen for the tert.-butyl substituted polymer $\mathbf{1 2 b}$.

The nonsubstituted paralinked structure $\mathbf{9 b}$ and the tert.butyl paralinked structure $\mathbf{1 2 b}$ both show a shoulder at about $350 \mathrm{~nm}$ which could be explained by some aggregation effects of the alkyl structure. None of these effects could be found in the DSC measurements.

Figure 6 shows the photoluminescence spectra of the metalinked polymers $\mathbf{9 a - 1 2 a}$ while Figure 7 shows the photoluminescence spectra for the paralinked polymers $9 \mathbf{b}-$ 12b. For the photoluminescence spectra, the excitation wavelength for all polymers was $300 \mathrm{~nm}$. The emission maxima

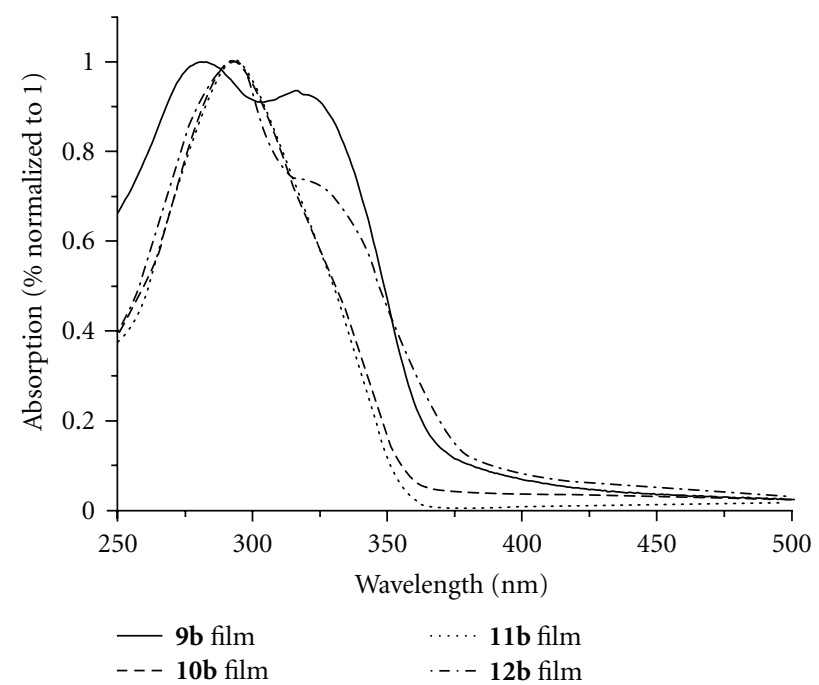

FIGURE 5: UV spectra of parasubstituted polymer films $\mathbf{9 b}-\mathbf{1 2 b}$ on silica glass.

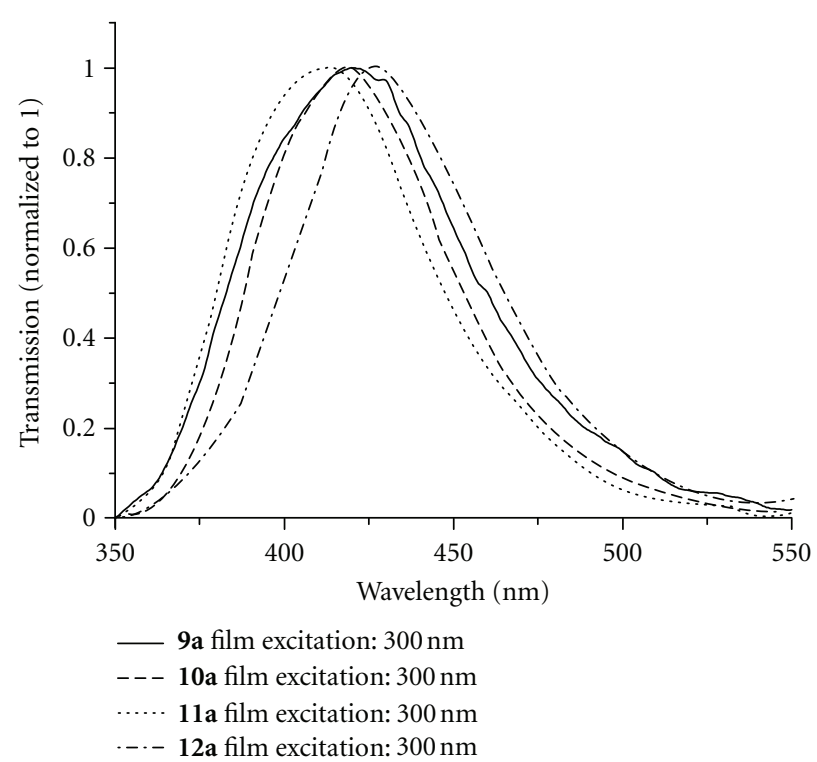

FIGURE 6: PL spectra of metapolymer films $\mathbf{9 a}-\mathbf{1 2 a}$ on silica glass. 


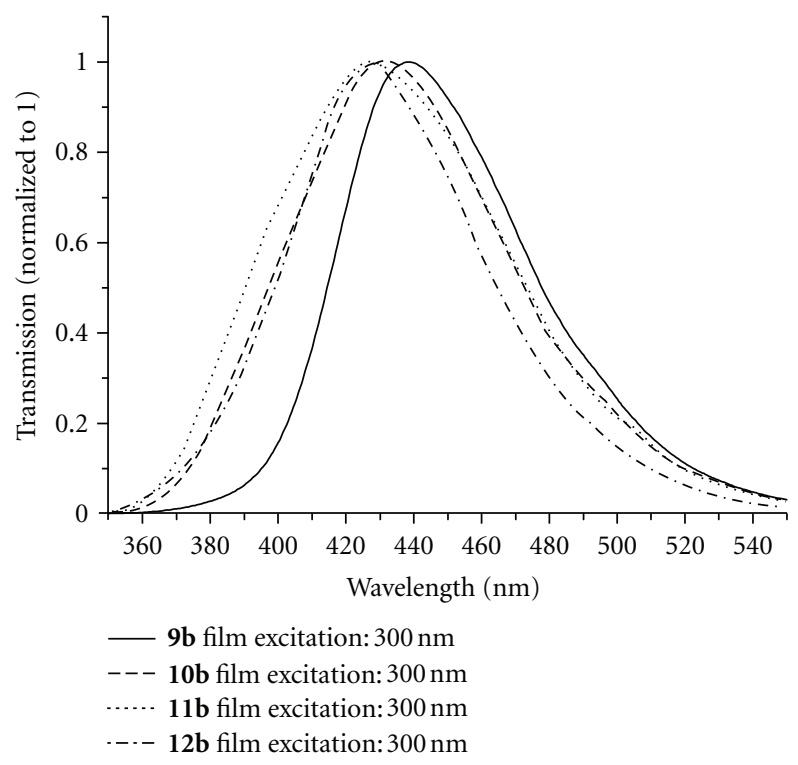

Figure 7: PL spectra of parapolymer films $\mathbf{9 b} \mathbf{- 1 2} \mathbf{b}$ on silica glass.

are seen between $420 \mathrm{~nm}$ and $440 \mathrm{~nm}$. The paralinked substituted polymers $\mathbf{9 b} \mathbf{b} \mathbf{- 1 2} \mathbf{b}$ show a higher emission wavelength maximum than their metalinked analogues. The highest difference between meta- and paralinked polymers of $18 \mathrm{~nm}$ is found for the nonsubstituted polymers $\mathbf{9 a}$ and $\mathbf{9 b}$. With increasing alkyl substitution length these differences become smaller. In the case of tert.-butyl substituted polymers 12a and $\mathbf{1 2 b}$ there is no significant deflection anymore.

3.3. HOMO and LUMO Energy Levels. Table 3 shows an overview of the reduction potentials as well as the resultant LUMO energy levels. Since the oxidation potentials for the synthesized polymers all lie above $+2 \mathrm{~V}$, in regard to their expected HOMO levels that should lie below $-6 \mathrm{eV}$, it was not possible to determine their HOMO levels with the $\mathrm{CV}$ equipment. Measurements in different solvents showed that the standard electrolyte was becoming oxidized before an oxidation of the polymer occurred. First, reduction behavior of the new polymers was investigated by applying the polymers as films on glass carbon electrodes. But in this case the ground electrolyte was reduced before the polymer or with the polymer, so that an exact determination of the LUMO levels was not possible. Therefore, CV measurements were carried out in dimethylformamide solutions with an extended reduction window to higher voltages of about $+2.5 \mathrm{~V}$. Because the polymers were not soluble in the used solvent $\mathrm{N}, \mathrm{N}$-dimethylformamide for $\mathrm{CV}$ measurements, their corresponding monomers were used instead. As Zeng et al. showed CV measurements for tris-phenyl-s-triazine finding a LUMO of $-2,6 \mathrm{eV}$ one can assume that the styryl group does not affect the LUMO level [17]. According to this, we estimated the monomer LUMO levels and equated them with the polymer LUMO levels in first approximation.

Figures 8 and 9 show the $1 \mathrm{e}^{-}$transfer found in the $\mathrm{CV}$ measurement. While Figure 8 shows the metalinked monomers, Figure 9 shows the results for the paralinked
TABLE 3: LUMO energy levels and reduction potentials of corresponding monomers $\mathbf{5 a} / \mathbf{b}-\mathbf{8} \mathbf{a} / \mathbf{b}$.

\begin{tabular}{lcc}
\hline Monomer & Reduction peak potential $^{1}(\mathrm{~V})$ & $\mathrm{LUMO}^{2}(\mathrm{eV})$ \\
\hline $\mathbf{5 a}$ & $-2,1$ & $-2,7$ \\
$\mathbf{5 b}$ & $-2,1$ & $-2,7$ \\
$\mathbf{6 b}$ & $-2,1$ & $-2,7$ \\
$\mathbf{7 a}$ & $-2,2$ & $-2,6$ \\
$\mathbf{7 b}$ & $-2,1$ & $-2,7$ \\
$\mathbf{8 a}$ & $-2,2$ & $-2,6$ \\
$\mathbf{8 b}$ & $-2,1$ & $-2,7$ \\
\hline
\end{tabular}

${ }^{1}$ Peak potential converted to $\left[\mathrm{Fc} / \mathrm{Fc}^{+}\right]$by adding $0,522 \mathrm{~V}$.

${ }^{2} \mathrm{LUMO}$ layer determined for $\left[\mathrm{Fc} / \mathrm{Fc}^{+}\right]$by adding $+4,8 \mathrm{eV}$.

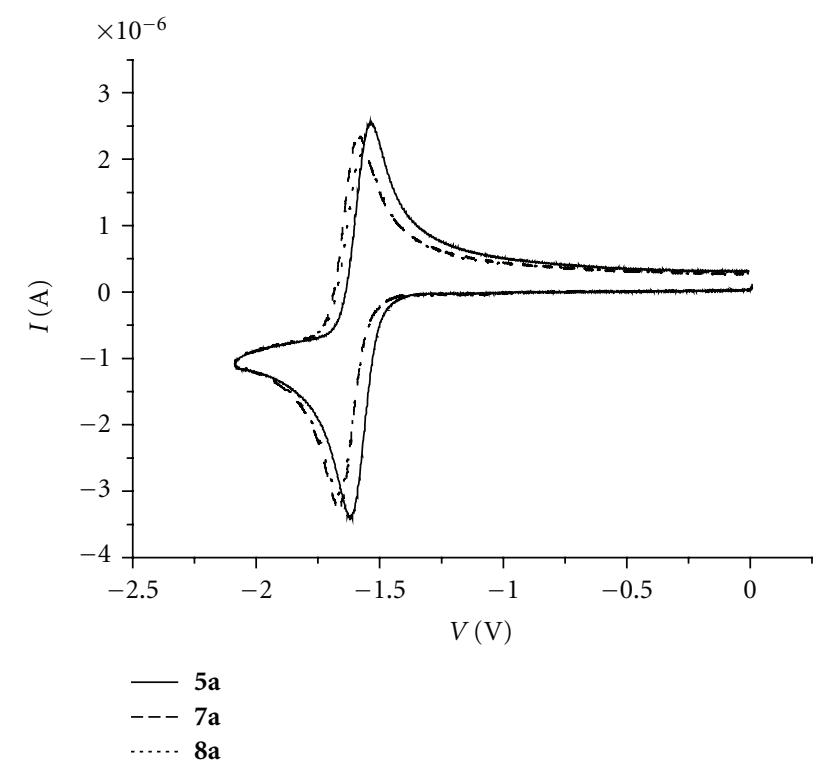

FIgURE 8: Cyclovoltammograms of monomers $\mathbf{5 a}-\mathbf{8 a}$ with $1 \mathrm{e}^{-}$ transfer (reduction cycles).

monomers. Figures 8 and 9 show the first reduction peak measured up to $-2,0 \mathrm{~V}$ which is completely reversible. The reduction potential for the metalinked monomers $\mathbf{5 a}-$ 8a is lower (about $0,05-0,07 \mathrm{~V}$ ) than their corresponding paralinked analogues $\mathbf{5 b}-\mathbf{8 b}$. The reoxidation potential shows a more pronounced difference between metalinked monomers (at about $-1,6 \mathrm{~V}$ ) and paralinked (at about $-1,9 \mathrm{~V})$ monomers. The LUMO values can be estimated from the reduction peak potentials to around $-2,6--2,7 \mathrm{eV}$, which should allow a quite efficient electron injection from the barium cathode into the emissive layer.

The whole reduction with the $2 \mathrm{e}^{-}$transfer for the metalinked monomers is shown in Figure 10 and for the paralinked monomers in Figure 11. It is seen that the second reduction step (at $-2,4 \mathrm{~V}$ ) is nonreversible for the metalinked monomers $\mathbf{5 a}-\mathbf{8} \mathbf{a}$. For these monomers a degradation product peak is seen in the reoxidation at about $-0,5 \mathrm{~V}$. In contrast to that the second reduction step (at $-2,1 \mathrm{~V}$ ) is nearly reversible for the paralinked monomers 


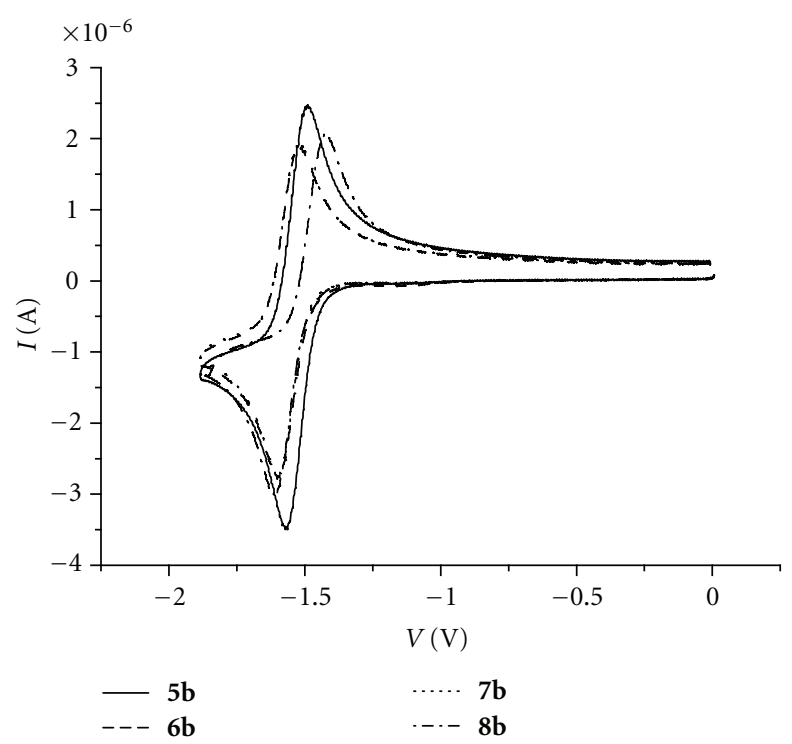

Figure 9: Cyclovoltammograms of monomers $\mathbf{5 b}-\mathbf{8} \mathbf{b}$ with $1 \mathrm{e}^{-}$ transfer (reduction cycles).

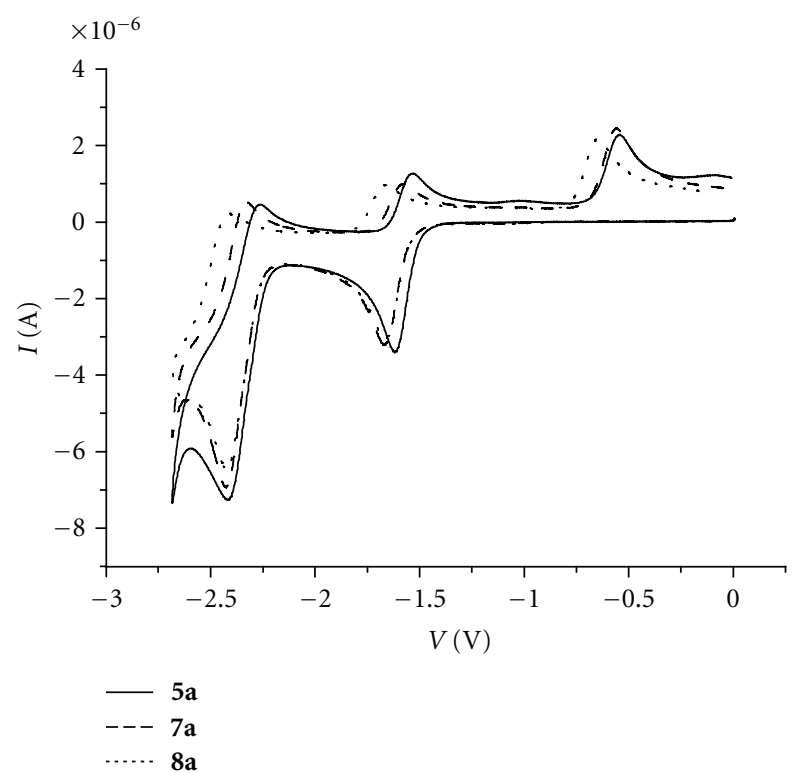

Figure 10: Cyclovoltammograms of monomers $\mathbf{5 a - 8 a}$ with $1 \mathrm{e}^{-}$and $2 \mathrm{e}^{-}$transfer (reduction cycles).

$\mathbf{5 b}-\mathbf{8 b}$. Summarizing, all monomers and, in first approximation, their corresponding polymers also exhibit a reversible first reduction step at $-2.1--2,2 \mathrm{~V}$. The second reduction step is only reversible for the paralinked monomers, whereas the metalinked monomers show nonreversibility during the second reduction step.

3.4. OLED Design. Figure 12 shows the OLED stack used for testing the new electron-transporting polymers $\mathbf{9 a} / \mathbf{b}-\mathbf{1 2} \mathbf{a} / \mathbf{b}$.

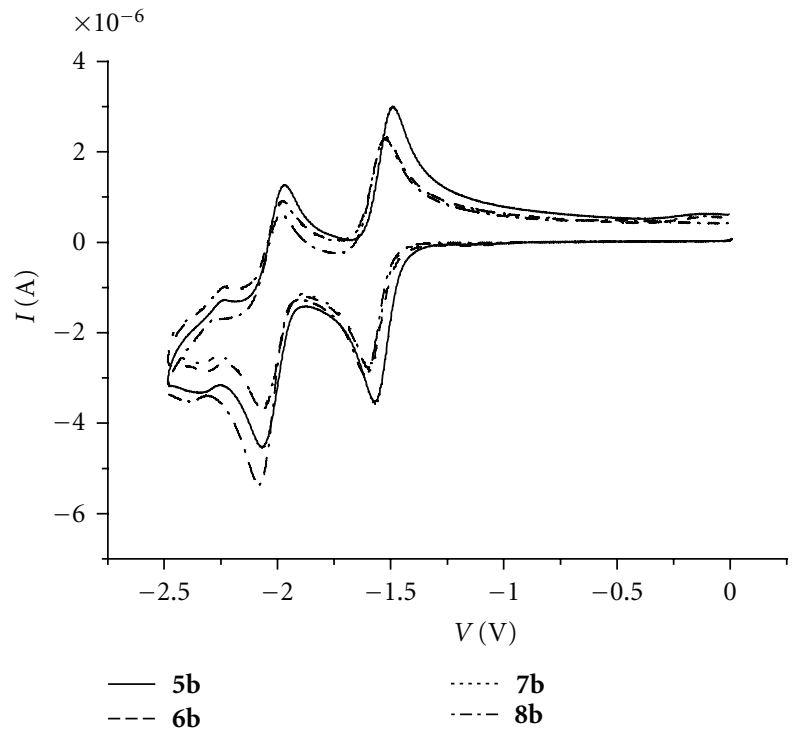

Figure 11: Cyclovoltammograms of monomers $\mathbf{5 b}-\mathbf{8} \mathbf{b}$ with $1 \mathrm{e}^{-}$ and $2 \mathrm{e}^{-}$transfer (reduction cycles).

All the blend components (polymers $9 \mathbf{9} / \mathbf{b}-\mathbf{1 2 a} / \mathbf{b} ; \mathbf{1 3}$; $\mathbf{C o H}-$ 001) (41.7 wt \%; $16.7 \mathrm{wt} \%$; $41.7 \mathrm{wt} \%)$ were tested in a green single emitting layer.

As a transparent anode material, a glass substrate coated with indium-tin-oxide from Optrex Europe $\mathrm{GmbH}$ was used. As hole injection layer PEDOT/PSS (Clevios CH8000 from Heraeus Holding $\mathrm{GmbH}$ ) was used. Furthermore a polymeric Interlayer 1 which is provided from Merck KGaA was deposited on top of the PEDOT. This layer improves hole transport, blocks electrons, and separates the phosphorescent-emitting layer from the PEDOT layer. The emitting layer is made out of a blend system of the new electron-transporting polymers $\mathbf{9 a} / \mathbf{b}-\mathbf{1 2} \mathbf{a} / \mathbf{b}$, the wideband gap cohost $\mathrm{CoH}-001$, and the emitter 13. Finally a metal cathode, consisting of barium/aluminium, was deposited in vacuum.

Figure 13 shows the different energy levels of the materials used in this OLED. The second hole injection layer, Interlayer 1 , has a $\mathrm{HOMO}$ energy level of $-5,1 \mathrm{eV}$ from which the holes are directly injected into the emitter molecule $\mathbf{1 3}$ with a HOMO energy level of $-5,1 \mathrm{eV}$. The electrons are injected from the inner barium cathode $(-2,5 \mathrm{eV})$ to the LUMO of the new polymers $\mathbf{9 a} / \mathbf{b}-\mathbf{1 2} \mathbf{a} / \mathbf{b}$ of about $-2,6 \mathrm{eV}$. The wideband gap material $\mathrm{CoH}-001$ exhibits a $\mathrm{HOMO}$ energy level of $-6,2 \mathrm{eV}$ and a LUMO energy level of $-1,8 \mathrm{eV}$. According to these energy levels, one can assume that $\mathrm{CoH}$ 001 does not contribute significantly to the charge transport in the blend system; however, it was found that it plays an important role to an optimized blend morphology which finally leads to an improved OLED performance.

In the following, we report the results obtained with the new electron-transporting polymers $\mathbf{9 a} / \mathbf{b}-\mathbf{1 2} \mathbf{a} / \mathbf{b}$ in the OLED stack and discuss them depending on the different polymer structures in regard to the alkyl substitution and to the kind of linkage to the polystyrene backbone. Figure 14 


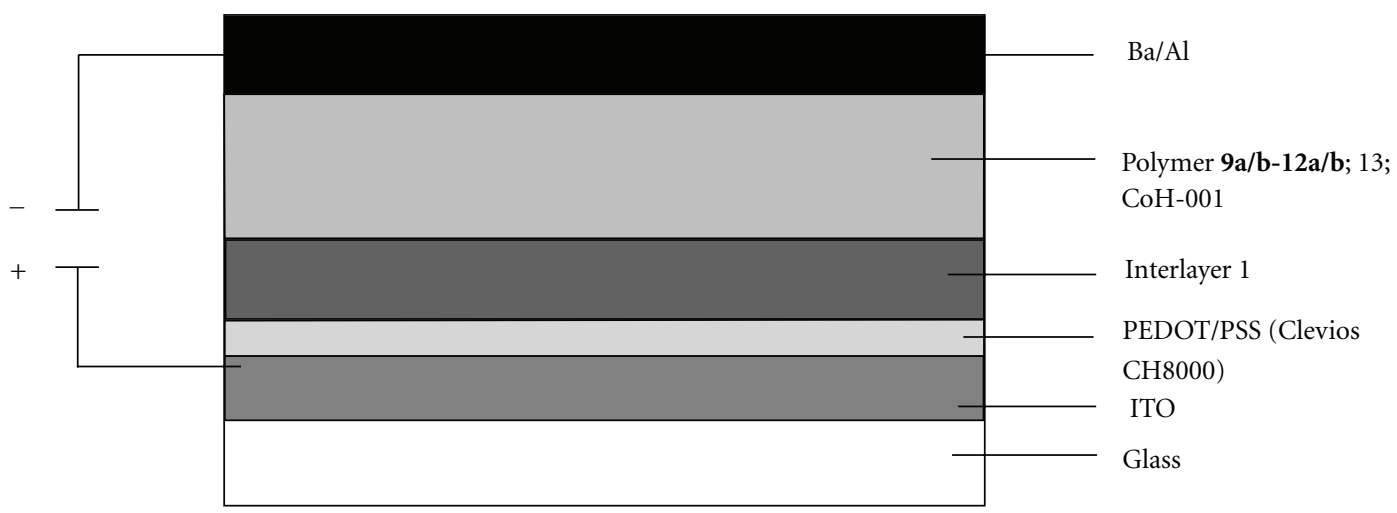

Figure 12: General OLED stack.

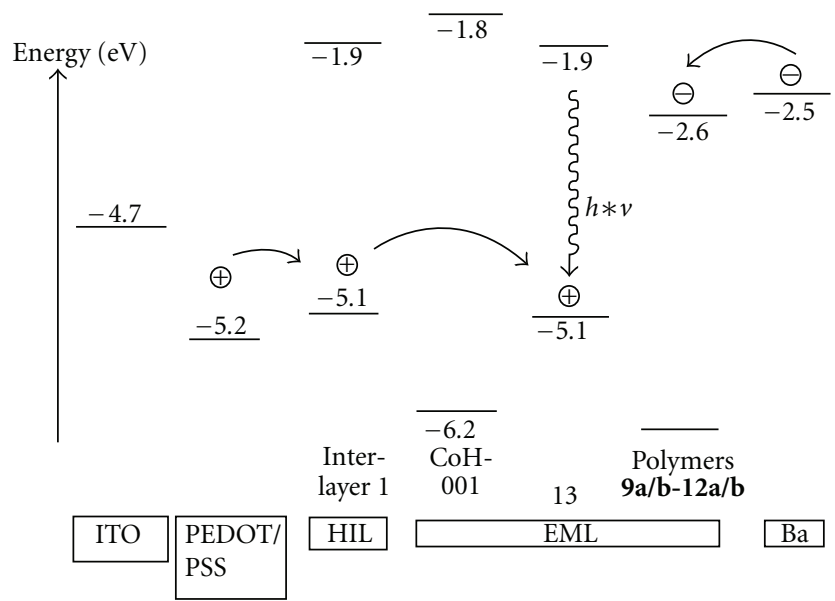

FIGURE 13: Energy levels of the used blend materials in the OLED stack.

shows the luminance-voltage plot for the OLEDs with the eight polymers $(\mathbf{9} \mathbf{a} / \mathbf{b}-\mathbf{1 2} \mathbf{a} / \mathbf{b})$ introduced in Table 2 . Figure 15 shows the corresponding current efficiencyluminance plot of the prepared OLEDs. The results obtained for the efficiency of the different polymers are listed in Tables 4 and 5.

Upon comparing the obtained luminances, we found that the metalinked structure $\mathbf{9 a - 1 2 a}$ always exhibits higher values than the corresponding paralinked polymers $\mathbf{9 b}-\mathbf{1 2} \mathbf{b}$ (Figure 14). Former studies on 1,3,5-triazinecore structures show that different shaped side groups affect the efficiency in an OLED device. Chen et al. studied different starshaped 1,3,5-triazinecore structures and the influence on side chains according to the electron mobility and triplet energy by using several green guests [12]. They found that 2,4,6-tris(biphenyl-3-yl)-1,3,5-triazine shows a high energy barrier at the interface. It has a low electron mobility, and as a result of this the hole and electron flow is better balanced. This leads to a high efficient device. The two other studied structures 2,4,6-tris(triphenyl-3-yl)1,3,5-triazine and 2,4,6-tris $\left(9,9^{\prime}\right.$-spirobifluorene-2-yl)-1,3,5triazine exhibit lower triplett energy and with this a lower efficiency. Related to our work one can assume that using

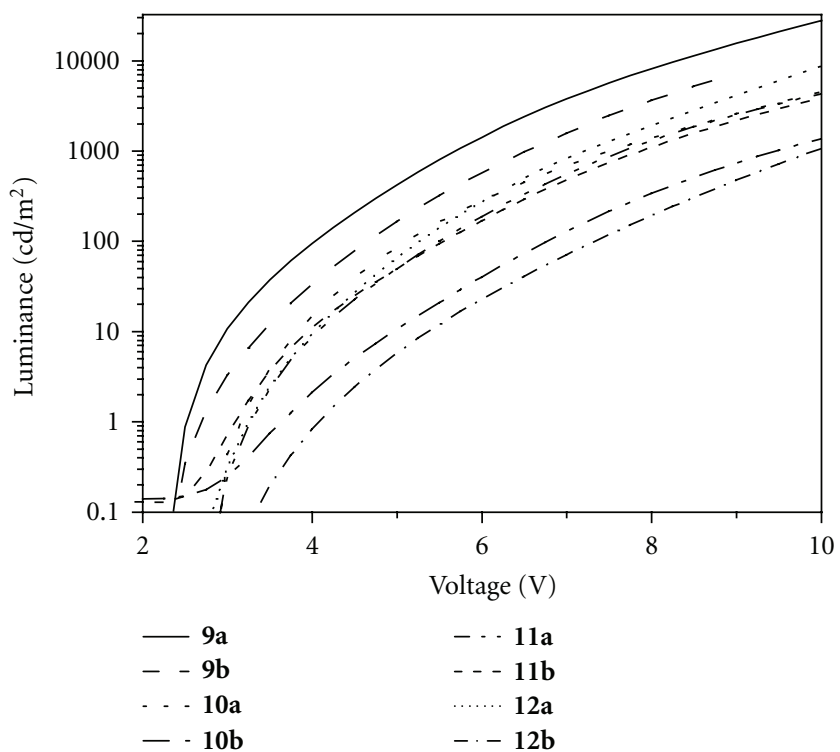

FIGURE 14: Luminance-voltage plot of OLEDs depending on the used electron-transporting polymers $\mathbf{9 a} / \mathbf{b}-\mathbf{1 2} \mathbf{a} / \mathbf{b}$.

metalinked phenyl-triazine polymers shows a better performance because of the improved triplett exciton limitation in the OLED stack.

In regard to the substitution $\left(R_{1}\right)$, the nonsubstituted polymers $\mathbf{9 a} / \mathbf{b}$ show better properties with luminances of more than $10.000 \mathrm{~cd} / \mathrm{m}^{2} @ 10 \mathrm{~V}$ and lowest voltage onsets of about $2,4 \mathrm{~V}$ compared to the alkyl-substituted polymers $\mathbf{1 0 a} / \mathbf{b}-\mathbf{1 2 a} / \mathbf{b}$. All substituted polymers $(10 a / b-12 a / b)$ show luminances between $800 \mathrm{~cd} / \mathrm{m}^{2}$ and $8000 \mathrm{~cd} / \mathrm{m}^{2} @ 10 \mathrm{~V}$. The onset voltage increases with alkyl substitution to values between 2,7-3,4 V.

According to the efficiency, there is a difference between meta- or paralinked polymers (Figure 15). The metalinked nonsubstituted polymer (9a) shows the best efficiency with $45 \mathrm{~cd} / \mathrm{A} @ 1080 \mathrm{~cd} / \mathrm{m}^{2}$ and is much higher than polymers with alkyl substitutions. Methyl, (10a) ethyl (11a) and tert.-butyl groups (12a) do not differ significantly in their performance. The efficiencies lie within the range of 34$35 \mathrm{~cd} / \mathrm{A} @ 5000 \mathrm{~cd} / \mathrm{m}^{2}$, and the luminances are lower than 
TABLe 4: Voltage and efficiency of metasubstituted polymers 9a-12a.

\begin{tabular}{lcccc}
\hline Device characteristics & 9a/TMM102+ TEG001 & 10a/TMM102 + TEG001 & 11a/TMM102 + TEG001 & 12a/TMM102 + TEG001 \\
\hline Voltage/V @ 100 cd/m & 4,4 & 5,35 & 5,1 & 5,25 \\
Voltage/V @ $1000 \mathrm{~cd} / \mathrm{m}^{2}$ & 6,25 & 7,75 & 7,5 & 7,1 \\
Voltage/V@ $5000 \mathrm{~cd} / \mathrm{m}^{2}$ & 8,2 & $>10$ & 10 & 9,1 \\
Voltage/V @ $10 \mathrm{~mA} / \mathrm{cm}^{2}$ & 7,7 & 9,5 & 9,25 & 8,5 \\
Luminance Efficiency/cd/A+ & $36 @ 8 \mathrm{~V}$ & $34 @ 8 \mathrm{~V}$ & $35 @ 7 \mathrm{~V}$ & $34 @ 8 \mathrm{~V}$ \\
\hline
\end{tabular}

TABle 5: Voltage and efficiency of parasubstituted polymers $\mathbf{9 b}-\mathbf{1 2} \mathbf{b}$.

\begin{tabular}{lcccc}
\hline Device characteristics & 9b/TMM102 + TEG001 & 10b/TMM102 + TEG001 & 11b/TMM102 + TEG001 & 12b/TMM102 + TEG001 \\
\hline Voltage/V @ $100 \mathrm{~cd} / \mathrm{m}^{2}$ & 4,85 & 6,75 & 5,5 & 6,8 \\
Voltage/V @ $1000 \mathrm{~cd} / \mathrm{m}^{2}$ & 6,85 & 9,5 & 8 & 9,1 \\
Voltage/V@ $5000 \mathrm{~cd} / \mathrm{m}^{2}$ & 9 & $>10$ & $>10$ & $>10$ \\
Voltage/V @ $10 \mathrm{~mA} / \mathrm{cm}^{2}$ & 7,85 & $>10$ & 9,5 & 10 \\
Luminance Efficiency/cd/A & $24 @ 7 \mathrm{~V}$ & $29 @ 10 \mathrm{~V}$ & $32 @ 8 \mathrm{~V}$ & $20 @ 10 \mathrm{~V}$ \\
\hline
\end{tabular}

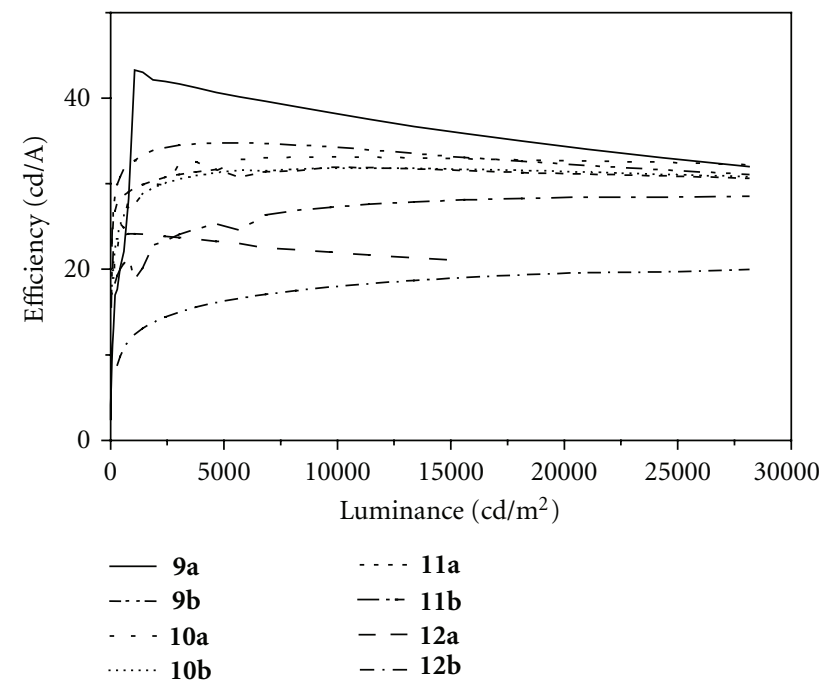

FIGURE 15: Current efficiency-luminance plot of OLEDs depending on the used electron-transporting polymers $9 \mathrm{a} / \mathbf{b}-\mathbf{1 2} \mathbf{a} / \mathbf{b}$.

for the nonsubstituted structure. No matter what kind of alkyl groups are added to the polymer, they show a lower performance in the OLED.

In contrast to the metalinked polymers, for paralinked polymers, the same difference cannot be found between alkyl-substituted $\mathbf{1 0 b}-\mathbf{1 2} \mathbf{b}$ and nonsubstituted polymers $\mathbf{9 b}$. The nonsubstituted structure (9b) has an efficiency of about $22 \mathrm{~cd} / \mathrm{A}$, more than $20 \mathrm{~cd} / \mathrm{A}$ lower than for the corresponding metalinked polymer 9a. Polymer 12b, with tert.-butyl groups, is the only one having lower efficiency. Methyl(10b) and ethyl-(11b) substituted polymers show higher efficiencies.

While comparing the performance of the metalinked triazine side groups and the paralinked side groups, better performance is found for all metalinked active side group polymers. Upon introduction of alkyl substituents, the performance of the OLEDs using these polymers as electron-transporting component generally is reduced. Alkyl substitution on the triazine polymer lowers the efficiency and luminance. The inserted side groups hinder an effective charge transport within the polymer structure. The longer or more compact the alkyl side group is, the smaller the reduction in the luminance.

The electroluminescence spectra in Figure 16 show a maximum absorption at about $520 \mathrm{~nm}$ and a small shoulder at $550 \mathrm{~nm}$ for all OLEDs using electron-transporting polymers $\mathbf{9 a} / \mathbf{b}-\mathbf{1 2} \mathbf{a} / \mathbf{b}$. All OLEDs produced with polymers $\mathbf{9 a} / \mathbf{b}-$ $\mathbf{1 2 a} / \mathbf{b}$ illustrate the same emission behavior which directly corresponds to the photoluminescence spectrum of the used emitter 13. No drift can be seen in the electroluminescence spectra; thus, there is no significant influence of the structure of the electron-transporting polymer used.

As a result of testing the new OLEDs depending on the synthesized polymers $9 \mathbf{a} / \mathbf{b}-\mathbf{1 2} \mathbf{a} / \mathbf{b}$, we found that the introduction of larger alkyl substitutions (ethyl, tert.-butyl) to the 2,4-diphenyl-1,3,5-triazine basic side-group structure leads to a significant loss in OLED performance. This can be explained by the electrical inactive alkyl chains which hinder an efficient charge transport in the blend. In contrast to that, the unsubstituted polymers $\mathbf{9 a} / \mathbf{b}$ already give good luminance and current efficiencies in single layer blend structures.

Besides the substituents, the choice of the linkage of the triazine basic structure to the polystyrene backbone is quite important for OLED performance. It is found that the metalinkage (9a-12a) leads to significantly enhanced luminance and current efficiencies in comparison to the para-linkage (9b-12b).

The general conclusion of this work is that a metalinkage of the active triazine unit to the polystyrene is preferred and that unsubstituted phenyl-triazines lead to most efficient devices. 


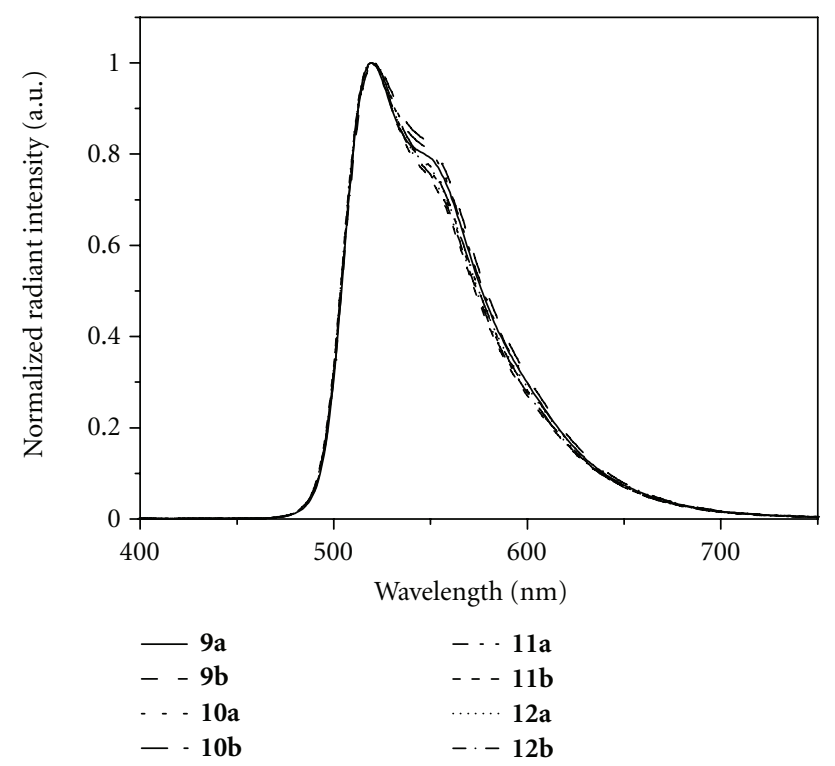

FIGURE 16: Electroluminescence spectra of OLEDs using different electron-transporting polymers $(\mathbf{9 a} / \mathbf{b}-\mathbf{1 2} \mathbf{a} / \mathbf{b})$ in the emitting blend.

\section{Conclusion}

Eight polystyrenes with electron-transporting 2,4,6-triphenyl-1,3,5-triazine side groups were synthesized. The triazine groups were linked in meta- or paraposition to the polystyrene backbone. Furthermore, the new polymers differ in alkyl substitution of the 4- and 6-phenyl rings. While comparing the meta- and the paralinked polymers, we recognize higher average molecular weight and higher glass transition temperatures for the paralinked polymers (10b-12b). The electron-transporting polymers were tested in green phosphorescent OLEDs in a blend with an Ir-emitter 13 and a cohost $\mathrm{CoH}-001$ as a wideband gap material.

The new electron-transporting polymers lead to efficient and highly luminescent OLEDs. The best performance was obtained for the polymers without alkyl substitution $\mathbf{9 a} / \mathbf{b}$. The alkyl substitution as an electrical insulating structure constrains an effective charge transport between the active species and finally leads to lower OLED efficiency and luminance. Furthermore, the metalinked polymers 9a-12a exhibit higher efficiency and luminance than their paralinked analogues $\mathbf{9 b} \mathbf{- 1 2 b}$.

Further studies will focus on copolymers consisting of the most promising electron-transporting candidates.

\section{Acknowledgments}

The authors would like to thank the Federal Ministry of Education and Research of Germany (BMBF) for the financial support within the NEMO project 13N10618. The authors would like to thank Stefanie Kreissl for device preparation.

\section{References}

[1] J. H. Burroughes, D. D. C. Bradley, A. R. Brown et al., "Lightemitting diodes based on conjugated polymers," Nature, vol. 347, no. 6293, pp. 539-541, 1990.

[2] T. R. Hebner, C. C. Wu, D. Marcy, M. H. Lu, and J. C. Sturm, "Ink-jet printing of doped polymers for organic light emitting devices," Applied Physics Letters, vol. 72, no. 5, pp. 519-521, 1998.

[3] N. B. McKeown, S. Badriya, M. Helliwell, and M. Shkunov, "The synthesis of robust, polymeric hole-transport materials from oligoarylamine substituted styrenes," Journal of Materials Chemistry, vol. 17, no. 20, pp. 2088-2094, 2007.

[4] C. Adachi, M. A. Baldo, M. E. Thompson, and S. R. Forrest, "Nearly $100 \%$ internal phosphorescence efficiency in an organic light emitting device," Journal of Applied Physics, vol. 90, no. 10, pp. 5048-5051, 2001.

[5] M. A. Baldo, M. E. Thompson, and S. R. Forrest, "Highefficiency fluorescent organic light-emitting devices using a phosphorescent sensitizer," Nature, vol. 403, no. 6771, pp. 750-753, 2000.

[6] B. Tong, Q. Mei, S. Wang, Y. Fang, Y. Meng, and B. Wang, "Nearly 100\% internal phosphorescence efficiency in a polymer light-emitting diode using a new iridium complex phosphor," Journal of Materials Chemistry, vol. 18, no. 14, pp. 1636-1639, 2008.

[7] M. Suzuki, S. Tokito, F. Sato et al., "Highly efficient polymer light-emitting devices using ambipolar phosphorescent polymers," Applied Physics Letters, vol. 86, no. 10, Article ID 103507, 3 pages, 2005.

[8] M. W. Thesen, B. Höfer, M. Debeaux et al., "Hole-transporting host-Polymer series consisting of triphenylamine basic structures for phosphorescent polymer light-Emitting diodes," Journal of Polymer Science, Part A, vol. 48, no. 15, pp. 34173430, 2010.

[9] M. W. Thesen, H. Krueger, S. Janietz, A. Wedel, and M. Graf, "Investigation of spacer influences in phosphorescentemitting nonconjugated PLED systems," Journal of Polymer Science, Part A, vol. 48, no. 2, pp. 389-402, 2010.

[10] M. Debeaux, M. W. Thesen, D. Schneidenbach et al., "Chargetransporting polymers based on phenylbenzoimidazole moieties," Advanced Functional Materials, vol. 20, no. 3, pp. 399408, 2010.

[11] C. W. Tang and S. A. Vanslyke, "Organic electroluminescent diodes," Applied Physics Letters, vol. 51, no. 12, article 913, 3 pages, 1987.

[12] H. F. Chen, S. J. Yang, Z. H. Tsai, W. Y. Hung, T. C. Wang, and K. T. Wong, "1,3,5-Triazine derivatives as new electron transport-type host materials for highly efficient green phosphorescent OLEDs," Journal of Materials Chemistry, vol. 19, no. 43, pp. 8112-8118, 2009.

[13] H. Inomata, K. Goushi, T. Masuko et al., "High-efficiency organic electrophosphorescent diodes using 1,3,5-triazine electron transport materials," Chemistry of Materials, vol. 16, no. 7, pp. 1285-1291, 2004.

[14] R. A. Klenkler, H. Aziz, A. Tran, Z. D. Popovic, and G. Xu, "High electron mobility triazine for lower driving voltage and higher efficiency organic light emitting devices," Organic Electronics, vol. 9, no. 3, pp. 285-290, 2008.

[15] M. Behl and R. Zentel, "Block copolymers build-up of electron and hole transport materials," Macromolecular Chemistry and Physics, vol. 205, no. 12, pp. 1633-1643, 2004. 
[16] M. Behl, E. Hattemer, M. Brehmer, and R. Zentel, “Tailored semiconducting polymers: living radical polymerization and NLO-functionalization of triphenylamines," Macromolecular Chemistry and Physics, vol. 203, no. 3, pp. 503-510, 2002.

[17] L. Zeng, T. Y. H. Lee, P. B. Merkel, and S. H. Chen, "A new class of non-conjugated bipolar hybrid hosts for phosphorescent organic light-emitting diodes," Journal of Materials Chemistry, vol. 19, no. 46, pp. 8772-8781, 2009. 

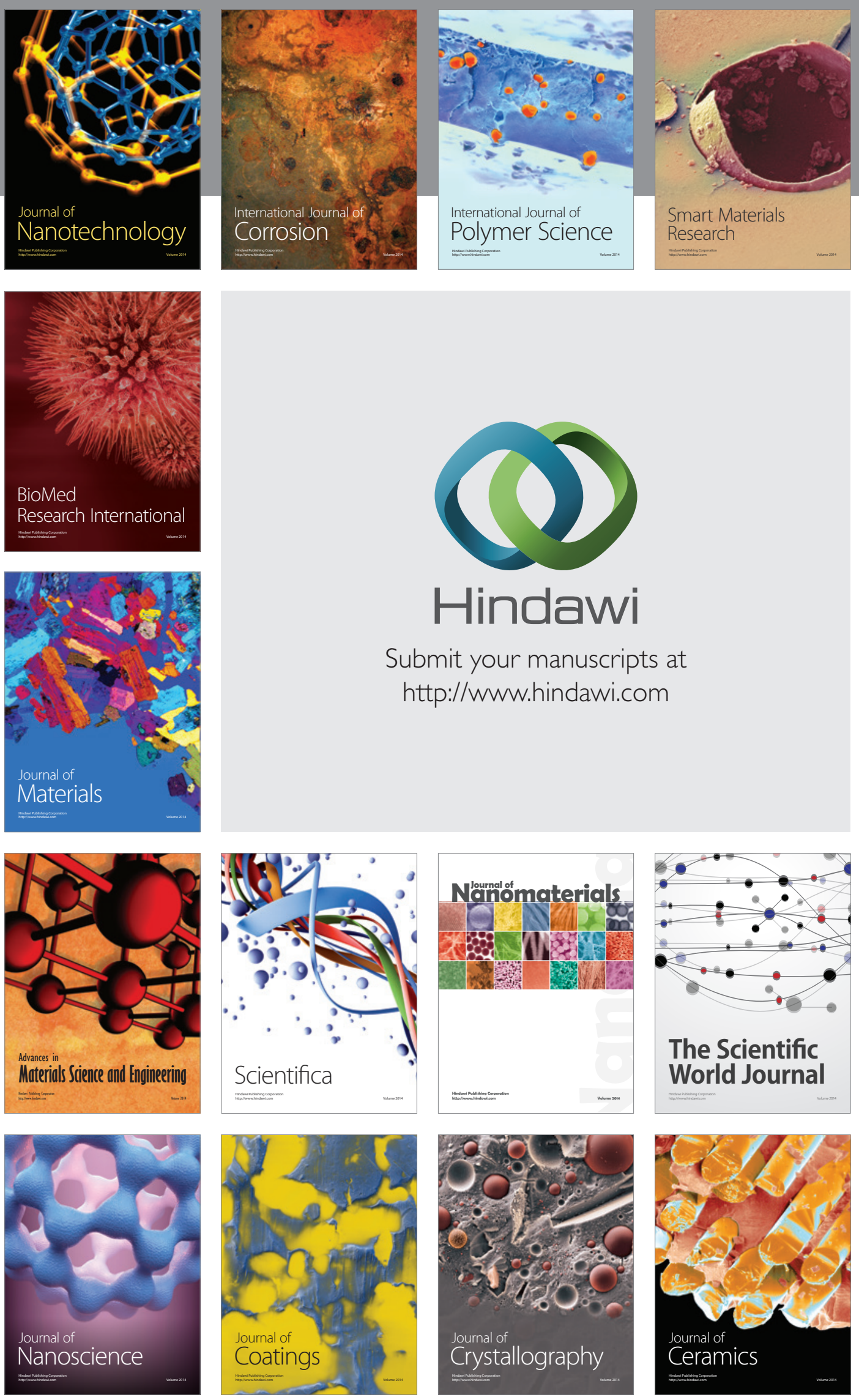

The Scientific World Journal

Submit your manuscripts at

http://www.hindawi.com

\section{World Journal}

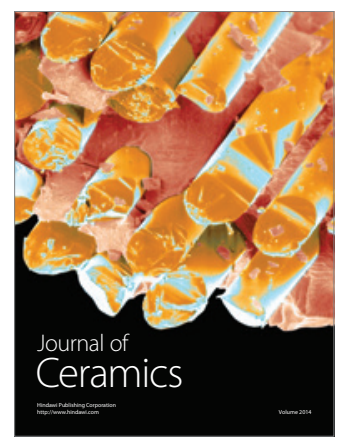

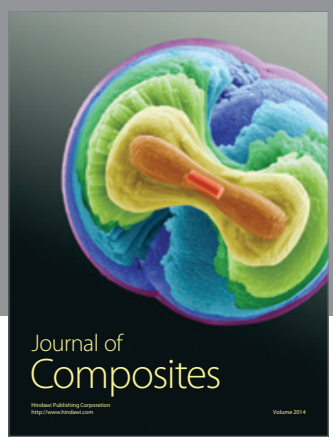
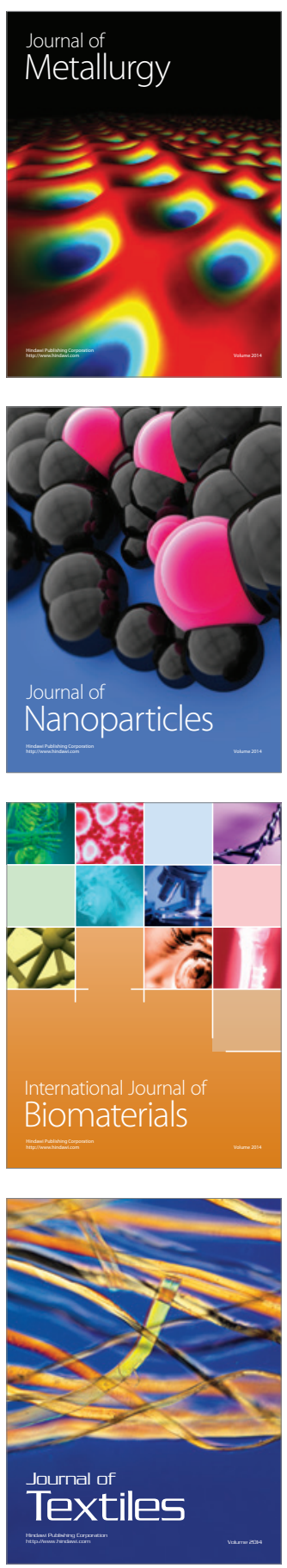